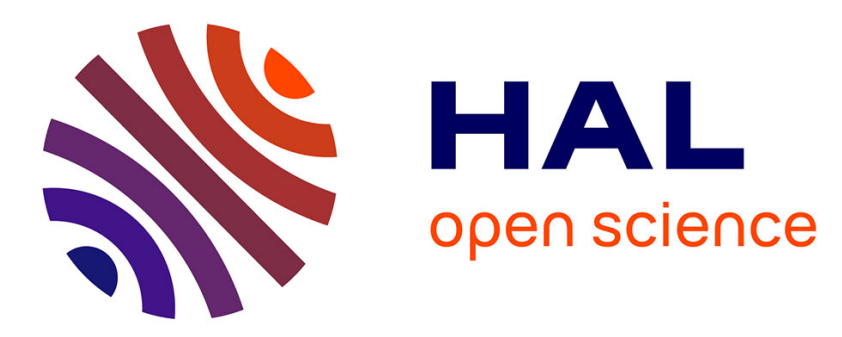

\title{
DNS of bypass transition in entrance channel flow induced by boundary layer interaction
}

Marc Buffat, Lionel Le Penven, Anne Cadiou, Julien Montagnier

\section{To cite this version:}

Marc Buffat, Lionel Le Penven, Anne Cadiou, Julien Montagnier. DNS of bypass transition in entrance channel flow induced by boundary layer interaction. European Journal of Mechanics - B/Fluids, 2014, 10.1016/j.euromechflu.2013.06.009 . hal-01264816

\section{HAL Id: hal-01264816 https://hal.science/hal-01264816}

Submitted on 29 Jan 2016

HAL is a multi-disciplinary open access archive for the deposit and dissemination of scientific research documents, whether they are published or not. The documents may come from teaching and research institutions in France or abroad, or from public or private research centers.
L'archive ouverte pluridisciplinaire HAL, est destinée au dépôt et à la diffusion de documents scientifiques de niveau recherche, publiés ou non, émanant des établissements d'enseignement et de recherche français ou étrangers, des laboratoires publics ou privés. 


\title{
DNS of bypass transition in entrance channel flow induced by boundary layer interaction
}

\author{
Marc Buffat*, Lionel Le Penven, Anne Cadiou and Julien Montagnier* \\ Laboratoire de Mécanique des Fluides et d'Acoustique LMFA, Université de Lyon, \\ Université Claude-Bernard Lyon 1/ CNRS/Ecole Centrale de Lyon/Insa de Lyon \\ 43, bd du 11 nov. 1918, 69622 Villeurbanne, FRANCE.
}

\begin{abstract}
Developing entrance flows are of interest in a large number of application areas. They have been widely studied in the past, but many aspects of these flows are not yet fully understood. One of the questions concerns the stability and turbulent transition in the entrance region of a channel. The present study investigates by direct numerical simulation (DNS) the laminar-turbulent transition induced by boundary layer interaction in a spatially developing flow near the entrance of a channel, where the laminar flow is linearly stable. For uniform inlet flow conditions and at sufficiently high Reynolds number, the turbulent transition actually takes place inside the boundary layers and well before the fully developed regime. The transition of one of the two boundary layers is triggered near the entry section by small amplitude perturbations, such as the ones created by small isolated obstacles distributed on the wall. These generate elongated streaks, and their transient growth and breakdown lead to the turbulent transition of the boundary layer near the entrance region. The transition of the second boundary layer is induced by the interaction with the first further downstream. After the transition of the second boundary layer, turbulence occupies the whole channel width and a turbulent channel flow develops. The transition is analyzed with the help of an orthogonal decomposition of the solenoidal velocity field. The observed transition scenario differs from existing simulations of streak breakdown where the perturbation is optimized for a transition to occur in a region far away from the entry section. In our simulations, the perturbation setup generates a significant non-linear growth of the streaks that are pushed away from the wall. Transient varicose instabilities,
\end{abstract}

*corresponding author

Email address: marc.buffat@univ-lyon1.fr (Marc Buffat) 
followed by sinuous instabilities induce the development of mushroom-shaped transitional structures in the outer region of the laminar boundary layer, that further break down downstream. The second boundary-layer transition resembles the one occurring under free-stream turbulence, with the generation of finite-length streaks and turbulent spots.

Keywords: bypass transition, boundary layer, channel entrance flow, DNS, orthogonal decomposition of solenoidal fields

\section{Introduction}

Despite many investigations since the famous Reynolds (1883) experiment, stability and turbulent transition in pipe and channel flows are still not well understood. In this context, most theoretical and numerical studies have focused on the parabolic Poiseuille solution, which is the laminar flow that can be observed far from the inlet, and less attention has been paid to the flow development in the entrance region. This question is however interesting in itself, since it has many engineering applications and also because any experimental work on fully developed Poiseuille flow is inevitably concerned with the effects of the disturbance of the incoming flow (Mullin [44]). At large Reynolds number, the laminar entrance flow in a plane channel corresponds to the development of two boundary layers, which far downstream merge to form the fully developed state. As shown by experimental, numerical and analytical studies (Shah and London [50],Durst et al. [26],Sadri and Floryan [47]), the distance required for flow development, also called the entry length $L_{e}$, varies linearly with the channel Reynolds number $R e=2 U_{0} h / v$ ( $h$ is the channel half-height and $U_{0}$ the flow rate velocity). If $R e$ is large enough, the value of $L_{e}$ (based on $99 \%$ of the Poiseuille centerline velocity $U_{m}$ ) is well approched by $L_{e} / h \simeq 0.08 R e$. On the other hand, linear stability theory predicts instabilities if $R e>R e_{c}^{\text {Pois }} \simeq \frac{4}{3} \times 5772=7696$ for the fully developed Poiseuille flow (Orszag [45], $U_{0}=\frac{2}{3} U_{m}$ ) and a critical Reynolds number $R e_{x_{c}}^{B L} \simeq 75000$ (based on the distance $x$ from the leading edge) for the Blasius boundary layer (Bertolotti et al. [6]). From this data, the distance $x_{c}$ at which the flow becomes linearly unstable, is estimated by $x_{c} / L_{e} \simeq 25 R e_{x_{c}}^{B L} / R e^{2}$, which is smaller than unity at large $R e$ and, in particular, for $R e>R e_{c}^{\text {Pois }}$. Thus, at high Reynolds number, the channel flow is linearly unstable in the entrance region. Indeed, the above analysis can only give a rough estimate of $x_{c}$ since the boundary layers are accelerated in the entrance region and differ from Blasius layers. Falkner-Skan boundary layers that are evolving in presence of streamwise pres- 
sure gradient are known to be more stable, in case of accelerated external flow (Schmid and Henningson [49],Criminale et al. [22]). The same stabilizing effect can be anticipated in the channel developing flow so that the previous estimation of $x_{c}$ is susceptible to be pushed away from the entry of the channel. One of the first stability analysis of the plane channel entrance flow is the study of Chen and Sparrow [18]. Using a basis flow solution obtained by the linearization method of Sparrow et al. [52] and parallel flow approximation, they show that the critical value of $R e$ decreases monotonically with increasing distance from the channel entrance, approaching the fully developed value $R e_{c}^{\text {Pois }}$ as a limit. Thus, depending on the Reynolds number, a large part of the entry zone can be linearly stable even for supercritical developed channel flow conditions. Despite this and thanks to our knowledge of turbulence transition in the boundary layers, it can be nevertheless be expected that the channel flow transition can occur for sub-critical conditions.

Indeed, in the presence of free-stream or wall perturbations, turbulent transition in boundary layers can occur under sub-critical conditions with respect to the linear stability theory (Durbin and $\mathrm{Wu}[25]$ ). In this case, the mechanism involving an exponentially growing mode is bypassed and transition is initiated by velocity perturbations taking the form of streaky structures elongated in the streamwise direction and modulated in the spanwise direction. Streaks are asymptotically decreasing perturbations in linear theory, but during the transient phase, they can exhibit large amplitudes capable of sustaining secondary instabilities evolving to turbulence. From a mathematical point of view, a first analysis of transient growth in Poiseuille flows was presented by Gustavsson [30], who invokes a resonance mechanism between Orr-Sommerfeld and Squire modes described in a previous paper (Gustavsson [31]). However, the described mechanism is quite different from that of the more recent transient growth studies (Trefethen et al. [53], Schmid and Henningson [49]), which may be regarded as being linked to the non-normality of the Orr-Sommerfeld operator. More recently, Zaki and Durbin [56] again invoke the resonance of an Orr-Sommerfeld and a Squire continuous mode to explain transient growth in the zero pressure gradient boundary layer. However, for channel flow, the eigenvalues of the Orr-Sommerfeld operator are countable and no continuous branch exists in the spectrum. In this case, by using the orthogonal decomposition presented in Section 3.1, Buffat and Le Penven [14] have proved that exact resonance of Orr-Sommerfeld and Squire modes are impossible and that transient growth results from a linear combination of a large number of non-orthogonal eigenmodes.

To study the effects of channel flow development on linear transient growth, 
Duck [24] has performed numerical simulations employing a parabolized version of the flow equations. Feeding the inlet flow with the growing eigenmode found by Luchini [37], he has shown that entry flow is susceptible to significant linear transient growth. For large spanwise wavelengths (comparable to the channel width) he has also demonstrated that this transient growth develops on a streamwise length scale of the order of $L_{e}$. Biau et al. [8] have also studied linear stability of the plane developping channel flow, adopting a basis flow characteristic of the far-downstream region and similar to the one used by Asai and Floryan [3]. Their modal analysis, conducted in the spatial framework with parallel-flow approximation, complements the temporal study made by Hifdi et al. [32]. Optimal transient perturbations have also been determined using the parabolized equations. As in the case of Falkner-Skan layers (Corbett and Bottaro [20],Tumin and Reshotko [54]), the accelerated free-stream appears to have a stabilizing effect on linear transient growth. However, it should be noted that this effect is progressive and that a capacity for significant amplification can still remains even though modal instability is completely suppressed by the effect of pressure gradient (Levin and Henningson [35]).

The focus of the paper is to describe and analyze numerical simulations of turbulent transition in a developing plane channel flow. Before undertaking such simulations, choices must be made regarding the perturbations to be introduced in the flow. For boundary layer flows, two methods are generally applied. One consists in introducing the perturbations randomly but consistently with some predefined spectrum (Jacobs and Durbin [34],Brandt et al. [12]), another is to select some special disturbances than can grow enough and initiate transition (Brandt and Henningson [11],Zaki and Durbin [56]). One way to do this is to select the perturbation that, according to linear approximation, yields to maximum amplification at certain distance downstream (Brandt and Henningson [11]). Generally, the inlet section is chosen at the leading edge and the target section is located at a relatively large distance (Luchini [38],Duck [24]). In a few studies, the inlet section is chosen at some distance from the entrance section, and in that case the perturbation growth rate is found to be larger (Levin and Henningson [35]). According to experimental studies (Fransson and Talamelli [28]), perturbations generated by isolated irregularities on the wall can also induce turbulent transition. These perturbations, usually localized inside the boundary layers, can be very effective in favoring earlier turbulent transition in a channel entrance flow. This reflection has inspired the method that is used in the following to perturb the channel flow.

To study turbulent transition at the entrance of a plane channel at high Reynolds 
number, we consider the development of boundary layers between two parallel walls. With the chosen parameters, the linear stability analysis indicates that the two laminar boundary layers can become exponentially unstable only in the last third of the studied domain. Thus, even if the chosen domain length is very large, a modal transition of the layers is unlikely to occur and turbulent transition is sub-critical. At the inlet, the boundary layer thickness is sufficiently small so that the boundary layers can be approximated to a Blasius boundary layer. The turbulent transition of one of the two boundary layers is triggered by a perturbation that models the perturbation generated by isolated wall obstacles near the entrance of the channel. This perturbation leads to a fast growth of stationary elongated streaks, that further break down and turbulent transition occurs. The transition of the second boundary layer is induced by the interaction with the first boundary layer further downstream. Direct numerical simulations of the NavierStokes equations are performed in such a configuration using a spectrally accurate method (Buffat et al. [15]). The configuration is elongated in the streamwise direction, so that the number of modes in the longitudinal direction is very large compared to the amount of nodes in the other directions. The simulation of such experiments typically requires billions of modes using a spectrally accurate approximation and should run on massively parallel high performance computers. To analyze the transition, an orthogonal decomposition of the solenoidal velocity fields is used on the disturbance flow. The observed transition scenario of the first boundary layer is different from previous analyses on the stability of steady streaks in ZPG boundary layers (Andersson et al. [2], Brandt et al. [10], Cossu et al. [21]) generated by free-stream turbulence because the chosen perturbation generated larger non-linear steady streaks. The observed mushroom-shaped structures are similar to those obtained by Bernard [5] in his boundary layer simulations using a hybrid vortex filament scheme.

This paper is organized as follows. In Section 2, the configuration of the flow at the entrance of the plane channel is presented, as well as the description of the laminar base flow and the perturbed flow. The numerical method and the analysis tools based on an orthogonal decomposition of solenoidal fields are described in Section 3. The inlet disturbance generation procedure is also discussed in this section. In Section 4, the DNS results are compared to the ZPG turbulent boundary layer case and to the fully-developed turbulent channel flow, to highlight the similarities and differences. The transition of both boundary layers are analyzed and discussed in Section 5, and the observed transition scenario is described. The concluding remarks are presented in Section 6 . 


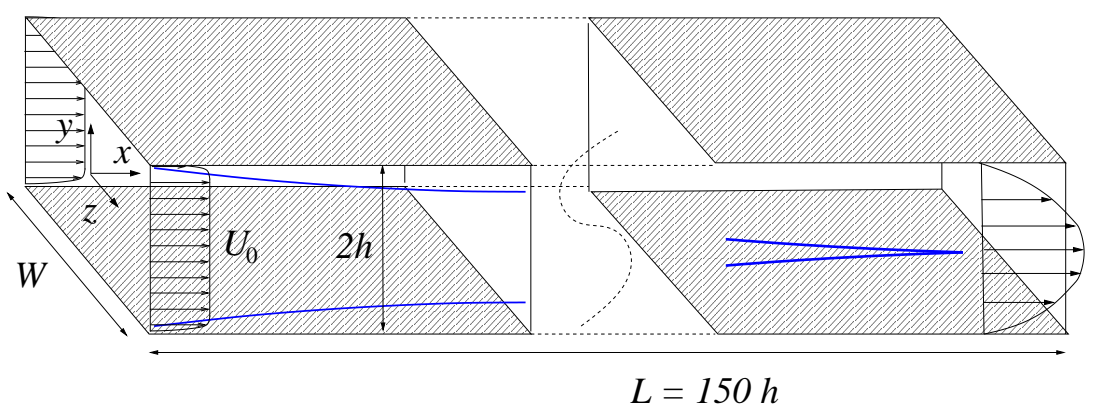

Figure 1: Domain of study $\Omega=\left[x_{0}, x_{0}+L\right] \times[-h,+h] \times[0, W]$

\section{Description of the problem}

We consider an incompressible flow at large Reynolds number, $R e=\frac{2 U_{0} h}{v}=$ 20000 ( $U_{0}$ is the mean velocity and $v$ is the viscosity), in the entrance region of a plane channel of width $2 h$ (see Figure 1). By assuming negligible perturbations at the entrance, the considered flow corresponds to the development of two boundary layers separated by a nearly inviscid core in the middle of the channel. In the downstream region, the boundary layers merge and the velocity profile reaches the profile of a developed channel flow. For laminar flow, the entrance length $L_{e}$ necessary to obtain the developed profile is proportional to the Reynolds number $\operatorname{Re}\left(\frac{L_{e}}{h} \approx 0.08 R e\right.$ in Durst et al. [26] $)$ and is very large at the studied Reynolds number $\left(L_{e} / h \approx 1600\right)$. If the boundary layers are turbulent, the entrance length $L_{e}$ is much smaller. Entrance lengths at least 10 times smaller are observed in experimental investigations of the turbulent entrance channel flow (Lien et al. [36]).

To study the interaction of the boundary layers, the computational domain, $150 h \times 2 h \times 3.2 h$, is elongated in the streamwise direction, as shown in Figure 1 , The upper boundary layer is perturbed at a distance $x_{0}$ from the entrance section. Experimental observations indicate a transition Reynolds number $R e_{x}=\frac{x U_{\max }}{v}$, based on the distance $x$ from the entrance and the maximum velocity $U_{\max }$ in the section ranging from $10^{5}$ (Roach and Brierley [46]) to $10^{6}$ (Matsubara and Alfredsson [40]) depending on the free-stream turbulence level. A local linear stability analysis of the Blasius boundary layer (Butler and Farrell [17]) indicates that the maximum transient energy growth increases according to $R e_{x_{0}}$ over a time period proportional to $\sqrt{R e_{x_{0}}}$. To induce a turbulent transition by a perturbation of small amplitude $(<1 \%)$ near the entrance, $x_{0}$ is chosen equal to $4 h$. The corresponding Reynolds number $R e_{x_{0}}=410^{4}$ is the same $R e_{x_{0}}$ as in Buffat et al. [15] , in which the transition is observed between $R e_{x}=1.510^{5}$ and $2.510^{5}$ depending 


\begin{tabular}{|c|c|c|c|c|}
\hline$X=\frac{x-x_{0}}{h}$ & 0 & 10 & 100 & 140 \\
\hline \hline$\frac{U_{\max }}{U_{0}}$ & 1.035 & 1.062 & 1.165 & 1.201 \\
\hline$\frac{\delta_{0.99}}{h}$ & 0.10 & 0.18 & 0.43 & 0.50 \\
\hline$R e_{x}=\frac{x U_{\max }}{v}$ & $4.110^{4}$ & $1.510^{5}$ & $1.210^{6}$ & $1.710^{6}$ \\
\hline
\end{tabular}

Table 1: Characteristics of the laminar base flow at $R e=20000$ and $x_{0}=4 h$

on the amplitude of the inlet perturbation, at $x_{0}$. The inlet boundary layer thickness $\delta_{0.99} \approx h / 10$ corresponds to a Reynolds number $\operatorname{Re}_{\delta}=200$, based on the Blasius scale $\delta=\sqrt{v x_{0} / U_{0}}$, and the inlet velocity profile corresponds to a Blasius profile in each half-section. In the following sections, $X=\frac{x-x_{0}}{h}$ denotes the non-dimensional distance from the entrance section of the computational domain.

\subsection{Laminar base flow}

Without inlet perturbation, the flow remains laminar. As seen in Table 1, the Reynolds number $R e_{x}$ ranges from $4.110^{4}$ up to $1.710^{6}$. The velocity $U_{\max }$ increases by $20 \%$ because of the growth of the boundary layer thickness $\delta_{0.99}$ from $0.1 \mathrm{~h}$ to $0.5 \mathrm{~h}$. The longitudinal pressure gradient is small and induces a slight increase of the skin friction coefficient $C_{f}$ compared with the one of a laminar ZPG boundary layer as seen on Figure 2. Due to this favorable pressure gradient, the shape factor of both laminar boundary layers is lower than the Blasius one (2.5 instead of 2.59).

The small favorable pressure gradient has however a strong influence on the linear stability of the boundary layer. Extracting velocity profiles from the calculated laminar solution, we have determined the linear neutral stability curve by a local temporal stability analysis. A comparison with the linear neutral curve obtained using Blasius profiles in each half-section is shown on Figure 2. Although the characteristics of the boundary layers of the laminar solution are similar to the Blasius-like profiles, the slight difference in the velocity profiles has a strong influence on the linear stability curve. As seen on Figure 2, the laminar entrance flow is linearly stable up to $x / h \approx 80$ instead of $x / h \approx 9$ for the Blasius profiles. This later position corresponds to the classical critical Reynolds number $R e_{\delta}=300$ of a Blasius boundary layer (Bertolotti et al. [7]). The exponential amplification is also very small (the real part of the most unstable Tolmien-Schlichting mode reaches a value of $6.10^{-3} U_{0} / h$ at $x / h=140$ ), thus a much longer computational domain is required for this unstable TS-wave to grow exponentially (an energy growth of 10 would require an evolution time of 200 and a computational domain at least two 

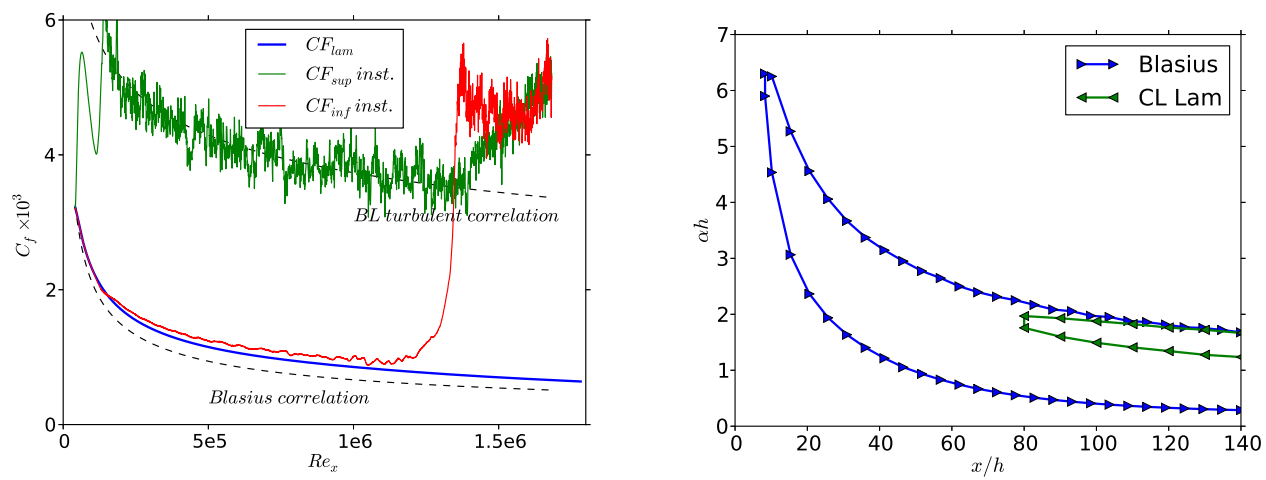

Figure 2: Base flow at $R e=20000$. a) Instantaneous skin friction coefficient for the laminar and perturbed case as a function of $R e_{x}$ compared to the Blasius and turbulent ZPG boundary layer correlation. b) Linear neutral stability curve in the channel compared with the Blasius one in the $\alpha h-x / h$ plane ( $\alpha$ is the streamwise wavenumber). 
times longer). Therefore, inside the present domain, a modal transition is unlikely to occur.

Transient energy growths have been calculated in the above configuration with the linear stability theory and they remain as large as in the ZPG boundary layer, indicating that even if the laminar flow is more stable than the Blasius boundary layer, bypass transition is not significantly delayed by the small favorable pressure gradient. Using the parabolized stability equations with a Falkner-Skan base flow at high Reynolds number, Levin and Henningson [35] also observed a large increase of the critical Reynolds number with a favorable pressure gradient. For a Hartree parameter $\beta_{H}=0.1$ similar to the estimated $\beta_{H} \approx 0.07$ in the present study, they found a critical Reynolds number $R e_{\delta}=889$ which is close to the critical Reynolds number obtained, $R e_{\delta} \approx 920$. They also found that, with a favorable pressure gradient base flow, the algebraic growth is much larger than the exponential growth, as in our configuration.

\subsection{Perturbed flow}

The upper laminar boundary layer is perturbed at the entrance of the channel by a perturbation of small amplitude $(<1 \%)$. This perturbation leads to a fast growth of elongated streaks in the near-wall region of the boundary layer and induces a rapid transition as seen on the instantaneous skin friction coefficient drawn on Figure 2, As the perturbation essentially acts inside the boundary layer, it can be experimentally generated by small obstacles on the wall as in Fransson and Talamelli [28]. An additional smaller random perturbation $(<0.1 \%)$ is superimposed on the first to trigger the breakdown of the nonlinear streaks. Turbulent transition of the upper boundary layer occurs near the entrance of the channel at $x / h \sim 10$. In contrast, no perturbation is imposed on the lower boundary layer, which remains laminar in a large part of the channel as seen on the skin friction coefficient in Figure 2, The turbulence developing in the upper boundary layer progressively acts on the lower boundary layer and the transition occurs only when the channel longitudinal length is sufficiently long $(>100 h)$. Near $x / h \sim 80$, low frequency fluctuations induced by the turbulent upper boundary layer interact with the laminar lower boundary layer and generate streaks. Then, non-linear breakdown of these streaks leads to a bypass transition of the lower boundary layer. Afterwards turbulence fills the whole channel width and a turbulent channel flow develops. Figure 3 depicts these main zones of interest from the value of the instantaneous longitudinal velocity in an arbitrary streamwise plane. 


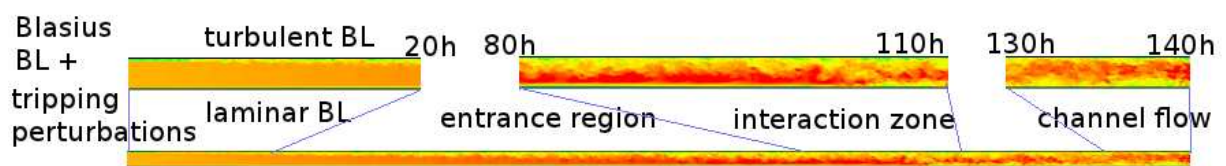

Figure 3: Perturbed flow: instantaneous longitudinal velocity in an arbitrary streamwise plane, with the main zones of interest: the entrance region, transition region and developed turbulent region at the end of the domain.

\section{Numerical method and analysis tool}

The numerical method and analysis of the results are based on a general orthogonal decomposition for solenoidal vector fields expressed in terms of projections of the velocity and vorticity fields on an arbitrary direction in space $\mathbf{e}_{y}$.

\subsection{Orthogonal decomposition of solenoidal fields}

For doubly-periodic flow with one direction of inhomogeneity $\mathbf{e}_{y}$ (the normal direction in Figure 1), Buffat et al. [15] derived an explicit form of this decomposition using the Helmholtz-Hodge theorem. Thus, any solenoidal velocity field is expanded in a Fourier series in the streamwise and spanwise directions in the form:

$$
\mathbf{u}(x, y, z, t)=\sum_{m=-\infty}^{\infty} \sum_{p=-\infty}^{\infty} \mathbf{u}^{m p}(y, t) e^{\boldsymbol{l}(\alpha x+\beta z)}
$$

where $\mathbf{u}^{m p}$ is the modal vector function of Fourier coefficients associated with wave-numbers $\alpha$ and $\beta$. Then, each Fourier component $\mathbf{u}^{m p}$ can be decomposed as $\mathbf{u}^{m p}=\mathbf{u}_{o s}^{m p}+\mathbf{u}_{s q}^{m p}$, where $\mathbf{u}_{o s}^{m p}$ and $\mathbf{u}_{s q}^{m p}$ are function of the normal velocity $v^{m p}(y)$ and normal vorticity $\omega^{m p}(y)$ Fourier component respectively :

$$
\mathbf{u}_{o s}^{m p}=\left(\boldsymbol{\imath} \frac{\alpha}{k^{2}} \partial_{y} v^{m p}, v^{m p}, \boldsymbol{\imath} \frac{\beta}{k^{2}} \partial_{y} v^{m p}\right)^{t}, \mathbf{u}_{s q}^{m p}=\left(-\boldsymbol{\imath} \frac{\beta}{k^{2}} \omega^{m p}, 0, \boldsymbol{\imath} \frac{\alpha}{k^{2}} \omega^{m p}\right)^{t}
$$

where $k^{2}=\alpha^{2}+\beta^{2}$. Both velocity and vorticity components exhibit orthogonal properties:

$$
\mathbf{u}_{o s}^{m p} \cdot \mathbf{u}_{s q}^{m p}=0 \text { and }\left(\mathscr{D}_{m p} \times \mathbf{u}_{o s}^{m p}\right) \cdot\left(\mathscr{D}_{m p} \times \mathbf{u}_{s q}^{m p}\right)=0
$$




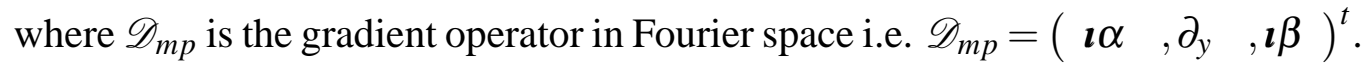
An $L_{2}$ orthogonal decomposition of the velocity field can easily be derived from this orthogonal decomposition of the Fourier components, such that :

$$
\mathbf{u}=\mathbf{u}_{s q}(v)+\mathbf{u}_{o s}(\omega) \text { with }<\mathbf{u}_{s q}, \mathbf{u}_{o s}>_{L_{2}}=0 \text { and } \nabla \cdot \mathbf{u}_{s q}=\nabla \cdot \mathbf{u}_{o s}=0
$$

The $\mathbf{u}_{o s}$ velocity field has zero wall-normal vorticity, its normal velocity is equal to the wall-normal velocity $v$ of $\mathbf{u}$ and its average in planes of constant $y$ coincide with the average of $\mathbf{u}$. The $\mathbf{u}_{s q}$ vector field has zero wall-normal velocity, its wall normal vorticity is equal to the wall-normal vorticity $\omega$ of $\mathbf{u}$ and its average in planes of constant $y$ vanishes. As the two velocity fields in the decomposition (4) are defined by the normal component of the velocity and the normal component of the vorticity respectively, these two fields $\mathbf{u}_{o s}$ and $\mathbf{u}_{s q}$ have been named in Buffat and Le Penven [14] as the Orr-Sommerfeld velocity (OS velocity) and Squire velocity (SQ velocity) fields, respectively.

\subsection{Numerical method}

The numerical method is based on the decomposition (4) applied to $\mathbf{u}$, the velocity field solution of the Navier-Stokes equations in a plane channel. The OS and SQ velocity fields are approximated using Fourier expansions in the $(x, z)$ planes with $M \times P$ modes and a Chebyshev polynomial expansion of degree $N$ in the $y$-direction as described in Buffat et al. [15]. In order to use Fourier expansion in the streamwise direction, the solution is made periodic in a larger domain using a fringe region (Bertolotti et al. [7]), where a forcing term is added to the NavierStokes equations to smoothly force the outflow to the prescribed inflow velocity field.

Using a Galerkin formulation of the incompressible Navier-Stokes equations, the unknown complex spectral coefficients of $\mathbf{u}_{o s}^{m p}$ and $\mathbf{u}_{s q}^{m p}$ are solutions of $M \times$ $P$ differential equations. They can be solved in parallel at each time step using a Crank-Nicholson/Adams-Bashforth scheme. Difficulties in the parallelization arise from the computation of nonlinear terms which require global datatransposition for the fast Fourier transforms. Using efficient 2D domain decomposition and hybrid parallel programming with MPI and high-level openMP threads (Montagnier et al. [42]), the "NadiaSpectral" computer code (Buffat [13]) runs efficiently on massively parallel computers (IBM BlueGene/P) using tens of thousands of cores for simulations with billions of modes. On such high performance parallel computers, typical simulation take 50 hours (elapsed time) using 16384 
cores. Otherwise these would have required 100 years on a mono-processor computer.

Two grid resolutions are used for the simulations: a medium grid with $11520 \times$ $128 \times 256$ modes and a refined grid with $17280 \times 192 \times 384$ modes. The value of $y^{+}$at the outlet is 0.16 for the medium grid, and 0.07 for the refined grid. Mean flow and statistics are similar for the two simulations, as seen in Figure 9. where the mean velocity results coincide for the two grids. A slight difference of $0.8 \%$ exists on the location of the second transition point due to the strong nonstationary nature of the transition, but this does not affect the main characteristics of the transition. The simulations can thus be considered as spatially resolved.

\subsection{Inlet perturbations}

Due to the convective instability of the boundary layer, perturbation needs to be continuously generated inside the computational domain to induce turbulent transition. In simulations, the most frequently-used procedure is the so-called recycling method, originally proposed by Lund et al. [39] and employed among others by Simens et al. [51]. Wu and Moin [55] periodically introduce a box of homogeneous turbulence above a laminar boundary layer at $\operatorname{Re}_{\theta}=80$. Schlatter et al. [48] use random volume forcing inside the laminar boundary layer at $R e_{\theta}=$ 180.

In our simulations, the laminar inlet profile of the upper boundary layer is altered by the optimal perturbation obtained from the local linear stability theory (Butler and Farrell [17]). The optimal perturbation is the initial disturbance experiencing maximum transient energy growth, calculated using the Orr-Sommerfeld/Squire equations and the entrance laminar profile. In experiments, this perturbation can be produced by a spanwise periodic array of small roughness elements fixed on the wall (Fransson et al. [27]) at a distance $x_{0}$ from the leading edge. This optimal perturbation consists of steady pairs of contra-rotating longitudinal vortices inside the boundary layer, with a spanwise wavenumber $\beta \approx \frac{2}{\delta}$ and zero streamwise wavenumber $\alpha$. With the orthogonal decomposition (1), it can be verified that this optimal perturbation is essentially an OS velocity field, as seen on Figure 4a. As explained in Buffat and Le Penven [14], this OS velocity perturbation induces a large linear transient growth and creates a large longitudinal SQ velocity field, that corresponds to steady streaks. This is illustrated on Figure 4a, where the linear response of this optimal mode consists mainly in an SQ velocity field.

In order to further characterize the steady streaks generated by this inlet perturbation, the streamwise evolution of the amplitude of the streaks is compared to the result obtained from the linear stability and to the result of Andersson et al. [2]. As 

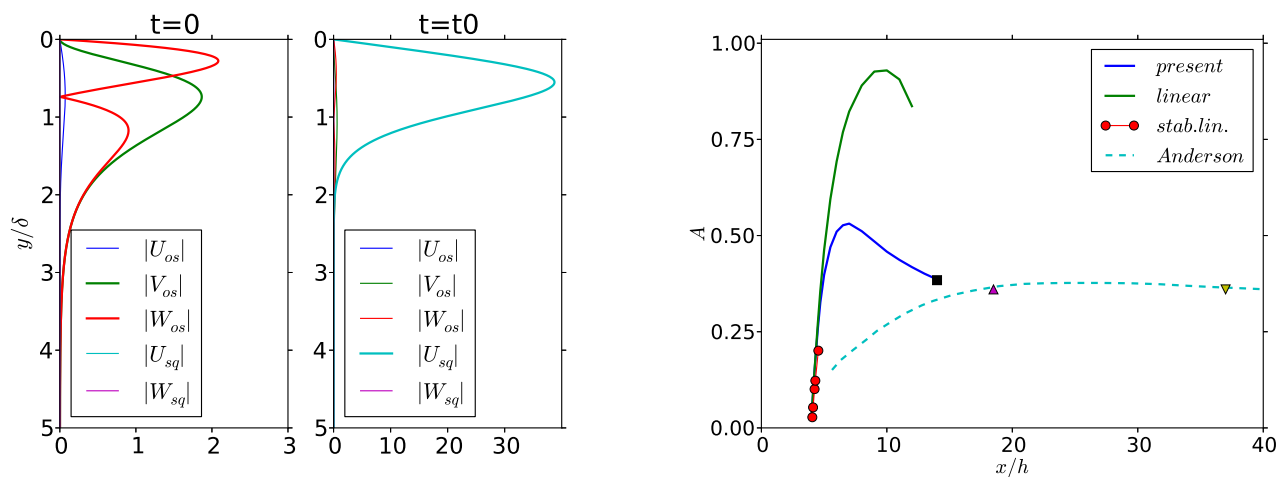

Figure 4: a) Orthogonal decomposition of the optimal perturbation at $t_{0}=0$ and its linear transient response at $t_{0}=t_{\max }$. b) Streak amplitude (as defined in Andersson et al. [2]) versus $R e_{x}$ compared to Andersson et al. [2] at $R e_{\delta_{0}}=430$. The circles represent the prediction using the local linear stability analysis. The symbol $\boldsymbol{\Delta}$ indicates the location $x=L$ of the output plane used in the spatial optimization of Andersson et al. [2] and the symbol $\boldsymbol{\nabla}$ the location $x=2 L$ of their stability analysis. The symbol $\mathbf{a}$ corresponds to the location of the transition studied in section 5.1

seen on Figure $4 \mathrm{~b}$, the inlet perturbation induces the development of fast growing streaks, followed by a viscous decay. However, without additional perturbation, transition is not observed. Using the laminar base flow described in section 2.1, the linear evolution of the perturbation is also shown on the figure, together with the result obtained with a local temporal stability analysis. The early development of the streaks coincides with the linear prediction, but later the non-linear interactions with the base flow limit their growth and their maximum amplitude reaches only half of the value of the linear streaks. The steady streaks obtained are also compared with those used by Andersson et al. [2] and later, by other authors (Hoepffner et al. [33] and Cossu et al. [21]) to study the stability of ZPG boundary layer streaks. Those streaks were also obtained using an optimal perturbation, but with a different target. This corresponds to the optimal perturbation imposed at the leading edge, that maximizes the energy growth at a specified downstream location $x=L$. This optimal perturbation has been calculated by Luchini [38] and Andersson et al. [1] using the parabolized stability equations (PSE) at high Reynolds number. It is similar to the present perturbation but with a spanwise wavenumber corresponding to the boundary layer thickness at the control location point $x=L$. Andersson et al. [2] then used the resulting linear optimal streaks at $x=0.3 L$ with $R e_{L}=910^{5}$ as boundary conditions for DNS in order to study the nonlinear development of the streaks and their stability at $x=2 L$. As seen on Figure $4 \mathrm{~b}$, these streaks have a smaller amplitude and a much smaller local 
growth rate than in the present case because they have been obtained by a global optimization with a large distance $L \sim 20 h$, representative of the effect of leading edge perturbations on a boundary layer at high Reynolds number. On the contrary, we have used a local optimization to generate, near the entrance at $x=4 h$, streaks with larger growth rates. This is consistent with the global linear stability analysis of Levin and Henningson [35], who found that the initial position of the perturbation has a significant impact on the growth of the streaks calculated using PSE. To further visualize the streaks, contours of constant streamwise perturbation are plotted on Figure 5a and compared to the linear perturbation in the section $X=4$, which is the location of the maximum amplitude of the linear streaks. As seen on this figure, large low-speed streaks are pushed away from the wall whereas high-speed streaks are squeezed near the wall, and the non-linear disturbance is quite different from the linear disturbance, which exhibits a symmetrical pattern between low and high speed streaks.

\subsection{Breakdown of boundary layer streaks}

The boundary layer on the upper wall is perturbed at the entrance section (inlet of the computational domain) by the optimal perturbation $\mathbf{u}^{\text {opt }}$ described in the previous paragraph. It corresponds to the following RMS values inside the boundary layer: $\left|u^{o p t}\right|_{r m s}=\left|w^{\text {opt }}\right|_{r m s} \approx 0.02 U_{0}$. The resulting streaky boundary layer is destabilized as detailed hereafter. To trigger transition, an additional random vector noise $\mathbf{r}(x, y, z)$ with a very small amplitude is superimposed on the optimal perturbation, such that the inlet disturbance can be written:

$$
\mathbf{u}^{\prime}(y, z, t)=\mathbf{u}^{o p t}(y, z)+\mathbf{u}^{r}\left(x=x_{0}-U_{0} t, y, z\right) \text { with } \mathbf{u}^{r}(x, y, z)=\mathscr{P}(\mathbf{r}(x, y, z))
$$

where $\mathscr{P}$ is the projection operator in divergence-free space. Figure 5 b shows a typical profile of the spanwise inlet perturbation. In the inlet section, the turbulence intensity of the optimal perturbation is equal to $T u^{o p t} \approx 0.017 U_{0}$ in the upper boundary layer and is ten times smaller elsewhere. Using a smaller box with $L=15 h$, we study the breakdown of the resulting streaky boundary layer for different small-amplitude perturbations $\mathbf{u}^{r}$. At the inlet the additional turbulence intensity $T u^{r}$ ranges from 0 (no additional perturbation) to $0.005 U_{0}$ (30\% of $\left.T u^{o p t}\right)$.

In this section, the base flow $\mathbf{U}_{b}$ is the unperturbed laminar flow and $\mathbf{U}$ the instantaneous velocity field. We will consider space-averaged values $\overline{\boldsymbol{u}}(x)$ of the perturbation $\mathbf{u}=\mathbf{U}-\mathbf{U}_{b}$ in the upper boundary layer defined as follows: 

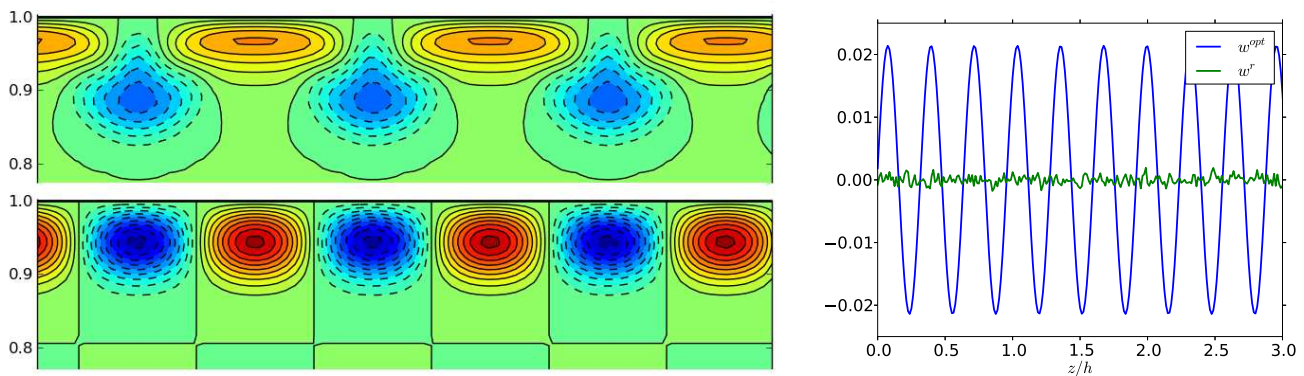

Figure 5: a) Comparison of the steady streak structure obtained with the optimal perturbation at $X=4$ from non-linear (top) and linear (bottom) simulation. The line display streamwise velocity perturbation with contour spacing $0.1 U_{0}$. Dashed lines are used for negative values. b) Instantaneous spanwise inlet primary perturbation $w^{o p t}$ and secondary perturbation $w^{r}$ at $y=0.9 h$ versus the spanwise coordinate $z / h$. 


$$
\overline{\boldsymbol{u}}(x)=\sqrt{\frac{1}{W \delta(x)} \int_{0}^{W} \int_{0}^{\delta} \boldsymbol{u}^{2} d z d y}
$$

Figure 6 shows the evolution of the streamwise primary disturbance $\bar{u}$ (the streaks) in the upper boundary layer. As seen on this figure, without a secondary perturbation, the streaks initially intensify downstream and then decrease by viscous dissipation. Using the orthogonal decomposition (1) on the disturbance $\overline{\mathbf{u}}$, the evolution of the contributions $\bar{u}_{O s}$ and $\bar{u}_{s q}$ is also plotted on the same figure. As explained in Buffat and Le Penven [14], in the linear phase the disturbance mainly consists of an SQ velocity $\bar{u}_{s q}$, which is characteristic of the linear transient growth. A little farther downstream, $\bar{u}_{s q}$ deviates from $\bar{u}$ due to non-linearities and an increase in the OS velocity $\bar{u}_{O S}$ is observed. In that region, the OS velocity field is independent of the spanwise direction and represents the nonlinear interactions of the streaks on the base flow. Further downstream, the two contributions $\bar{u}_{o s}$ and $\bar{u}_{s q}$ are of the same order of magnitude, indicating that the amplitude of the nonlinear interactions of the streaks on the base flow becomes as large as the amplitude of the streaks itself. In this streaky boundary layer, the sum of the laminar base flow $U_{b}$ and the OS velocity field $\bar{u}_{o s}$ represents the spanwise-averaged nonlinear mean flow and the SQ velocity field $\bar{u}_{s q}$ the streaky disturbance around this nonlinear mean flow. The profile of this nonlinear mean flow is drawn on Figure $7 \mathrm{~b}$ for different inlet amplitudes of the secondary perturbation. Compared to the laminar profile, the mean flow accelerates the perturbations in the near-wall region and slows it down in the outer border of the boundary layer. As a consequence, the low speed streaks are pushed away from the wall, as seen on the contour plots of the streamwise perturbation on Figure 5. We also observe the appearance of a strong inflection point in these mean profiles, that are linearly unstable according to Fjortoft's criterion (Schmid and Henningson [49]).

With random secondary perturbations $\left(T u^{r} \neq 0\right)$, streak breakdown is observed even for small-amplitude perturbations $\mathbf{u}^{r}$, as denoted by the oscillations of the streamwise disturbance observed on Figure 6 for $T u^{r}=0.1 \%, 0.25 \%$ and $0.5 \%$. In all these cases, the skin-friction coefficient increases up to the ZPG boundary layer turbulent correlation as seen on Figure 2 a for $T u^{r}=0.1 \%$, indicating a turbulent transition of the boundary layer. The growth of these secondary disturbances $\mathbf{u}^{\prime \prime}=\mathbf{U}\left(u^{r}\right)-\mathbf{U}\left(u^{r}=0\right)$ is depicted on Figure 6 $\mathrm{b}$, where the streamwise evolution of its instantaneous spanwise-averaged contributions $\bar{u}_{\text {" }}{ }_{o s}$ and $\bar{u}_{s q}{ }_{s q}$ are plotted for different inlet perturbations $\mathbf{u}^{r}$. As expected from the inflectional mean profiles, these secondary disturbances are initially amplified. As for the pri- 

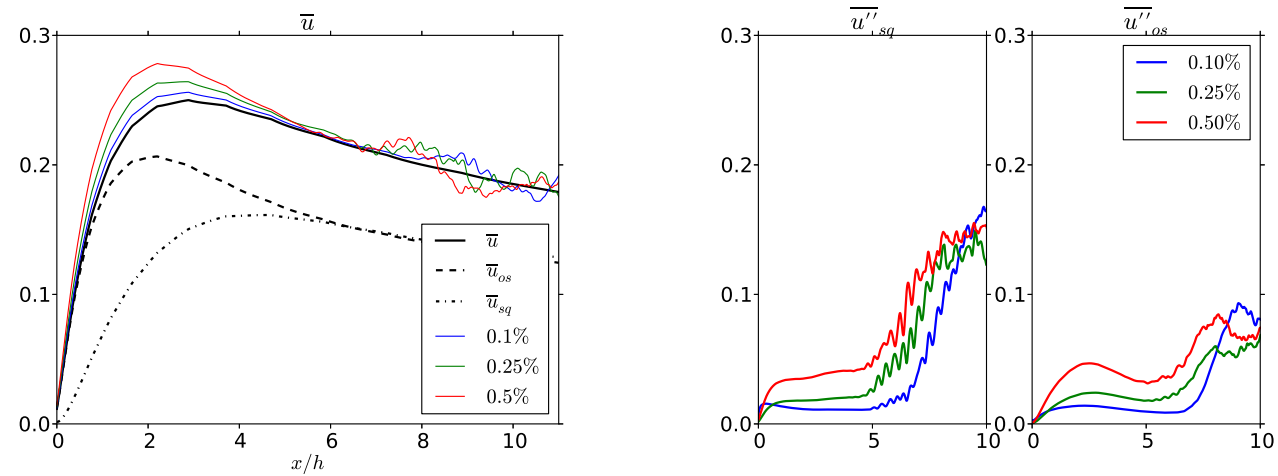

Figure 6: a) Streamwise evolution of the instantaneous spanwise-averaged streamwise disturbance (streaks) $\bar{u}(x, t)$ in the upper boundary layer obtained for an inlet primary perturbation $u^{o p t}$ with $\beta=2 / \delta, R_{\delta}=357$ and $T u^{o p t}=1.7 \%$ and different values of the secondary inlet perturbations $T u^{r}=0 . \%, 0.1 \%, 0.25 \%, 0.5 \%$. The dotted lines represent the two contributions $\bar{u}_{o s}$ and $\bar{u}_{s q}$ of the orthogonal decomposition (1) of $\bar{u}$ (i.e. $\bar{u}^{2}=\bar{u}_{o s}^{2}+\bar{u}_{s q}^{2}$ ) without secondary inlet perturbations $\left(T u^{r}=0 \%\right)$. b) Streamwise evolution of the secondary instantaneous spanwise-averaged streamwise disturbance $\overline{u^{\prime \prime}}(x, t)$, decomposed using (1) as ${\overline{u^{\prime \prime}}}^{2}={\overline{u_{o s}^{\prime \prime}}}^{2}+{\overline{u_{s q}^{\prime \prime}}}^{2}$, for different values of the secondary inlet perturbations $T u^{r}=0.1 \%, 0.25 \%, 0.5 \%$.

mary disturbance, the SQ velocity $u_{s q}^{\prime \prime}$ is a streaky disturbance, whereas the OS velocity $u_{o s}^{\prime \prime}$ models a nonlinear interaction with the mean flow. After this first growing phase, corresponding to the growth phase of the primary disturbance, $\overline{u_{o s}^{\prime \prime}}$ decreases slightly like the primary disturbance. Afterwards, a large increase of $\overline{u_{s q}^{\prime \prime}}$ is observed near $X=5$, followed by a similar increase of $\overline{u_{o s}^{\prime \prime}}$. The increase of $\overline{u_{s q}^{\prime \prime}}$ is characterized by streamwise periodic oscillations with a wavenumber identical to the spanwise wavenumber $\beta$ of the streaks. Then, near $X=10$, the profiles of $\overline{u_{o s}^{\prime \prime}}$ and $\overline{u_{s q}^{\prime \prime}}$ present a chaotic behavior, indicating a turbulent transition.

The reason for this secondary instability is an inviscid local mechanism caused by inflection points in the instantaneous velocity profiles. In the boundary layer, the perturbations can appear in the flow either in a spanwise symmetric (varicose) or antisymmetric (sinuous) pattern with respect to the underlying streak. If the instability occurs in the wall-normal direction, the secondary instability is a varicose mode, whereas if it appears in the transverse direction, it is a sinuous mode. In order to characterize the observed transition, Figure 7a shows the shape of the secondary disturbance compared to the contour lines of the streaky primary disturbance. As seen on this figure at $X=2$, the secondary disturbance has initially a varicose symmetry, which enhances the inflectional mean profile. Then, a sinuous 

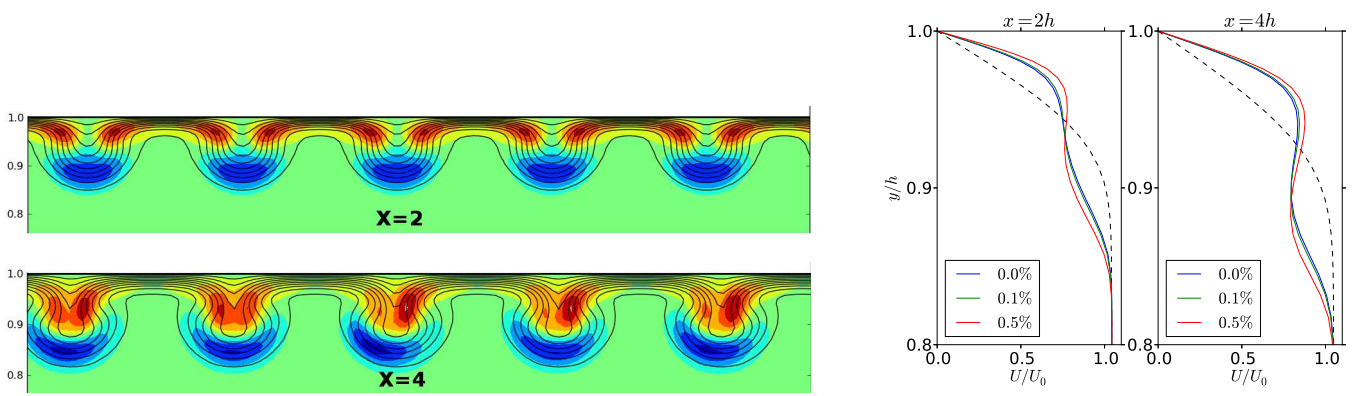

Figure 7: a) Contour lines $\left(0<u<U_{0}\right)$ in the $(z, y)$-plane at $X=2$ and $X=4$ of the streamwise velocity inside the upper streaky boundary layer obtained for a primary perturbation $u^{o p t}$ with $\beta=2 / \delta, R e_{\delta}=357$ and $T u^{o p t}=1.7 \%$ and without secondary perturbations $\left(T u_{r}=0\right)$. The isovalues in color represent the instantaneous values of the secondary disturbance $u^{\prime \prime}$ with $T u_{r}=$ $0.5 \%$ ( -0.17 (blue) $<u^{\prime \prime} / U_{0}<+0.17$ (red)). b) Instantaneous spanwise averaged profiles of the streamwise velocity $u$ at $X=2$ and $X=4$ for different secondary perturbations $T u_{r}$ compared with the laminar profile (dashed line).

instability occurs, characterized by spanwise oscillations of the head of the low speed streaks in the outer border of the boundary layer as seen at $X=4$ on Figure 7

\section{DNS results}

Starting from an initial laminar flow, the inlet perturbation described in Section 3.3. with $T u^{o p t} \approx 0.017 U_{0}$ and an additional random noise with $T u^{r} \approx 0.001 U_{0}$, is introduced at the inlet section. The turbulence level imposed at the entrance section is substantially smaller than the previous DNS of the ZPG boundary layer as in Wu and Moin [55] (Tu $\approx 6 \%)$ or in Brandt et al. [12] $(T u \approx 4.7 \%)$, showing the effectiveness of this inlet perturbation. The simulation first runs during a domain travel time $\tau_{L}=L / U_{0}$ in order to establish the mean flow described in Section 2.2. Then, statistical quantities are accumulated over time during another domain travel time $\tau_{L}$. The velocity field $u$ is averaged over time and space in the spanwise direction to obtain the mean velocity $U$. The reference velocity for the boundary layer profiles is the external velocity, chosen as the maximum mean velocity in the section.

\subsection{Integral properties}

The evolution of the mean skin-friction coefficient $C_{f}$ is shown on Figure 8 a for both walls. On the upper wall, the coefficient $C_{f}$ quickly increases, then decreases to settle near the ZPG boundary layer turbulent correlation $C_{f}=0.059 \operatorname{Re}_{x}^{-0.2}$. 

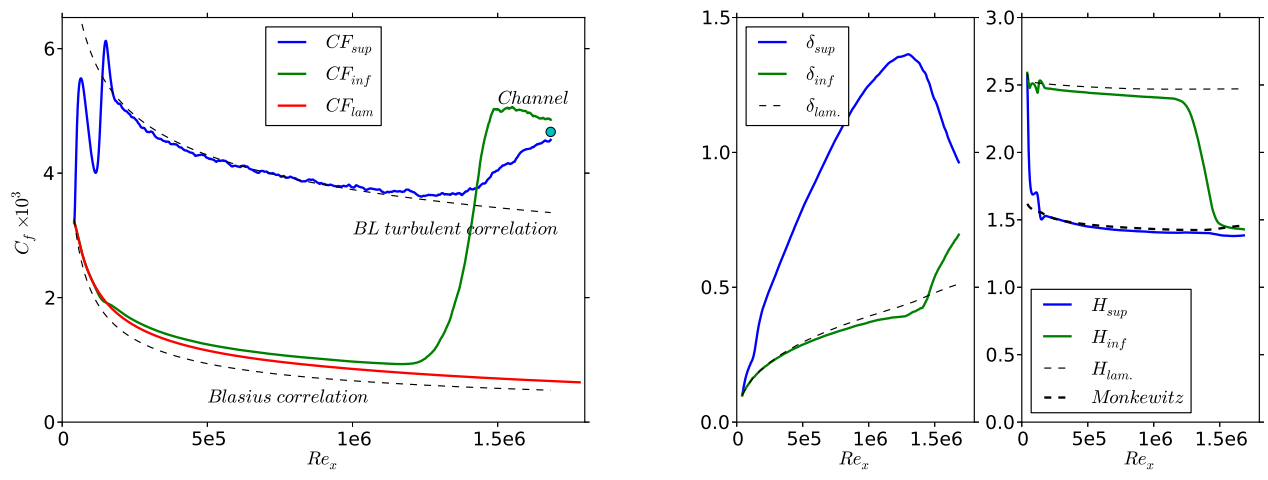

Figure 8: Perturbed flow: a) mean skin-friction coefficient $C_{f}$ and b) boundary layer thickness $\delta / h$ and shape factor $H$ as a function of the Reynolds number $R e_{x}$, compared to the laminar solution, the Blasius solution and turbulent ZPG boundary layer correlation.

The first step is characteristic of the nonlinear growth of the streaks (up to $R e_{x}=$ $6.10^{4}$ ), which induces a transient secondary inflectional instability, followed by a viscous decay of the streaks. Between $\operatorname{Re}_{x}=10^{5}$ and $1.610^{5}$, the large increase of $C_{f}$ characterizes the turbulent transition. The first overshoot of $C_{f}$ as a result of bypass transition, is also present in ZPG boundary layer simulations (Schlatter et al. [48]). On the lower wall, $C_{f}$ follows the laminar correlation, with a slight increase near $R e_{x}=10^{5}$ due to the transition of the upper boundary layer. A turbulent transition is observed further downstream between $R e_{x}=10^{6}$ and $1.210^{6}$. This transition induces an increase of the coefficient on the lower and upper wall, and both values of $C_{f}$ tend towards the fully-developed turbulent channel flow correlation $C_{f}=0.073\left(\frac{U_{0}}{U_{\max }}\right)^{2} R e^{-0.25}$ (Dean [23]). On Figure 8 $\mathrm{b}$, the boundary layer thickness $\delta$ of the upper wall exhibits fast growth. It reaches $68 \%$ of the channel width at the location of the transition of the lower boundary layer. On the lower wall, the boundary layer thickness first follows the laminar correlation, and then abruptly increases after transition. The evolution of the boundary layer shape factor $H=\frac{\delta^{*}}{\theta^{*}}$ is shown on the same figure and is compared to the laminar value $H=2.5$ and the asymptotic correlation given by Monkewitz et al. [41] for a ZPG turbulent boundary layer. As can be seen, the integral characteristics of the turbulent upper boundary layer are close to those of a ZPG turbulent boundary layer up to the transition of the lower boundary layer. 

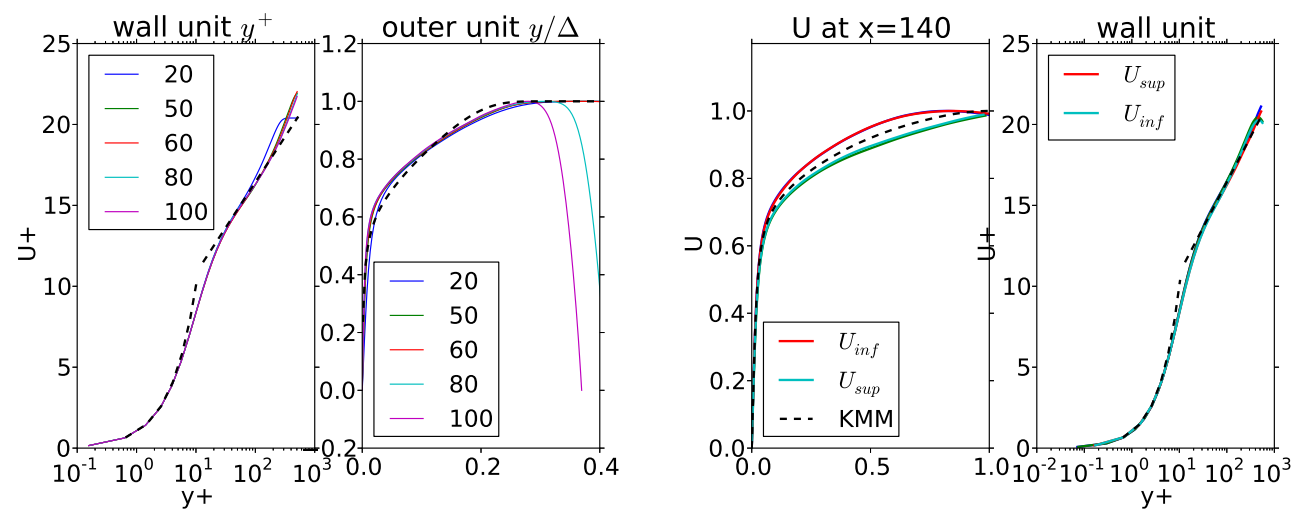

Figure 9: a) Mean velocity profile in the upper boundary layer in the wall unit and outer unit at different sections $X$ (before the transition of the lower boundary layer) compared to the logarithmic law (with $\kappa=0.41$ ) and the asymptotic behavior of [41] for a ZPG boundary layer. b) Mean velocity profile at the outlet section $X=140$ compared to the profiles in a turbulent channel flow at similar $R e_{\tau}=590$ [43]. The results obtained with the two grids are plotted on the same figure and the curves coincide.

\subsection{Mean velocity profiles}

Figure 9 (a) depicts the inner-scale and outer-scale mean profiles in the upper turbulent boundary layer at different sections before the second transition point. As can be seen, the profiles are self-similar in the viscous sublayer and log-law region and they follow the self-similar viscous logarithmic law of the wall. In the outer region, using the Rotta-Clauser length scale $\Delta=\delta^{*} U_{\infty} / U_{f}$, the profiles are also self-similar, but differ significantly from the asymptotic behavior of Monkewitz et al. [41] due to the favorable pressure gradient.

Figure 9 shows the mean profile in the outlet section $X=140$ compared to the DNS results in a developed turbulent channel flow at a similar $R e_{\tau}$ (Moser et al. [43]). As seen on this figure, the profiles are not very different from the mean velocity profile of a fully-developed turbulent channel flow, indicating that the domain length $L$ is of the order of the entrance length $L_{e}$.

\section{3. $R M S$ values}

The RMS values are obtained from the time-averaged values and spatiallyaveraged values in the transverse direction. On Figure 10, we show the evolution of the mean turbulent intensity $T u=\sqrt{\left(\left\langle u^{2}\right\rangle+\left\langle v^{2}\right\rangle+\left\langle w^{2}\right\rangle\right) / 3}$ in three regions of the channel: the upper boundary layer $\left(T u_{u p}\right)$, the lower boundary layer $\left(T u_{l o w}\right)$ and in between $\left(T u_{\text {mid }}\right)$. These averaged values are defined by the following: 


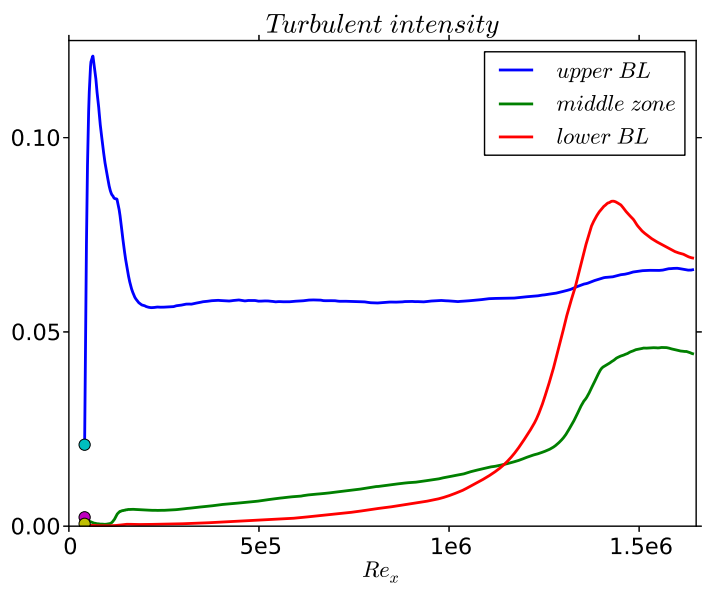

Figure 10: Streamwise evolution of the mean turbulent intensity $T u_{u p}$ in the upper and $T u_{l o w}$ in the lower boundary layers, and $T u_{\text {mid }}$ in between.

$$
\begin{gathered}
T u_{u p}(x)=\frac{1}{W \delta_{\text {sup }}(x)} \int_{0}^{W} \int_{h-\delta_{u p}}^{h} T u d z d y, T u_{\text {low }}(x)=\frac{1}{W \delta_{\text {low }}(x)} \int_{0}^{W} \int_{-h}^{-h+\delta_{\text {low }}} T u d z d y, \\
T u_{\text {mid }}(x)=\frac{1}{W} \int_{0}^{W} \int_{-h+\delta_{\text {low }}}^{h-\delta_{\text {sup }}} T u d z d y
\end{gathered}
$$

As can be seen on this figure, the turbulent intensity, $T u_{u p}$, grows very rapidly from a low inlet value of $2 \%$ up to a large value of $12 \%$ at $X=2$ and then decreases to a nearly constant value of $6 \%$. This reflects the fast growth of the streaks till $X=2$, followed first by a viscous decay till $X=8$, then by a destabilization that leads to transition near $X=10$. We also observe a low level (less than $1.5 \%$ ) of the turbulent intensity $T u$ inside the lower boundary layer and in between the two boundary layers before the second transition point $(60<X<100)$.

The mean RMS values of the turbulent intensity are summarized in table 2 before the second transition point at $X \approx 100$. From these values, we observe that the turbulent intensity $T u_{\text {low }}$ in the lower boundary starts to increase significantly near $X \approx 60$, when the turbulence level $T u_{\text {mid }}$ in between the two boundary layers (i.e. the free stream turbulence) reaches about $1 \%$. Further downstream near $X \approx 100$, the transition is characterized by a fast rise of the turbulence level $T u_{\text {low }}$, that reaches the level of turbulence $T u_{u p}$ in the upper boundary layer, as seen on Figure 10, 


\begin{tabular}{|c|c|c|c|c|}
\hline$X$ & 40 & 60 & 80 & 100 \\
\hline \hline$T u_{u p}$ & 0.06 & 0.06 & 0.06 & 0.06 \\
\hline$T u_{\text {mid }}$ & 0.006 & 0.009 & 0.012 & 0.019 \\
\hline$T u_{\text {low }}$ & 0.001 & 0.003 & 0.007 & 0.032 \\
\hline
\end{tabular}

Table 2: Mean RMS values of the turbulent intensity in different sections before the second transition point at $X \approx 100$.
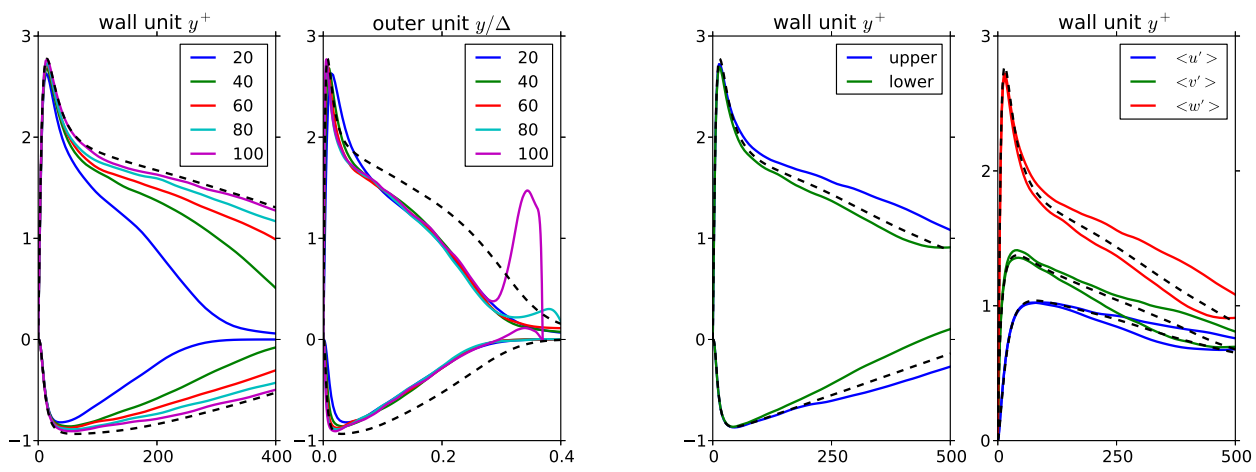

Figure 11: a) RMS profiles $\left\langle u_{+}^{2}\right\rangle^{1 / 2}$ and $\left\langle u v_{+}\right\rangle$in the upper turbulent boundary layer in the wall unit $y^{+}$and outer unit $y / \Delta$ at different sections $X$ before the transition of the lower boundary layer, compared to the ZPG boundary layer profiles at a similar $R e_{\theta}=2000$ of [48] (dotted line). b) RMS profiles $\left.\left\langle u_{+}^{2}\right\rangle^{1 / 2},<u v_{+}\right\rangle,\left\langle v_{+}^{2}\right\rangle^{1 / 2},\left\langle w_{+}^{2}\right\rangle^{1 / 2}$ in the outlet section $X=140$ compared to the profiles in a turbulent channel flow at a similar $R e_{\tau}=590$ [43] (dotted line).

On Figure 11, $\left.u_{+}^{2}\right\rangle^{1 / 2}$ and $\left\langle u v_{+}\right\rangle$in the upper turbulent boundary layer in the wall unit $y^{+}$and outer unit $y / \Delta$ at different $X$ locations before the second transition point $(X<100)$. In the wall unit, the profiles are similar to those in a turbulent ZPG boundary layer, as confirmed by comparison with profiles at a similar $R e_{\theta}=2000$ from the DNS of Schlatter et al. [48]. In the outer unit, using the Rotta-Clauser length scale $\Delta$, the profiles are self-similar in the outer region, but differ from the ZPG boundary layer due to the favorable pressure gradient. On Figure $11 \mathrm{~b}$, the Reynolds stresses are plotted in the outlet section $(X=140)$ and compared to the classical results of Moser et al. [43] for a fully developed turbulent channel flow. 


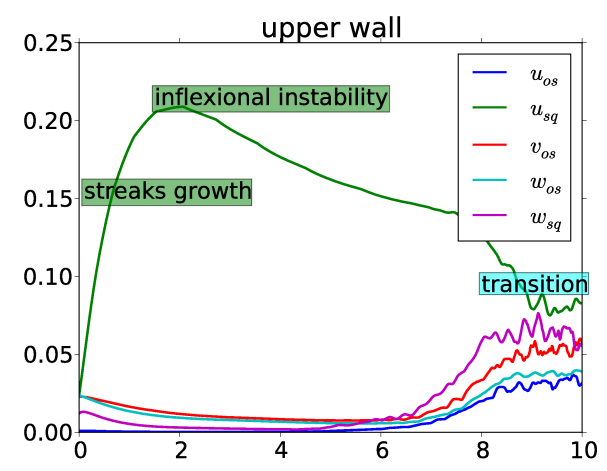

Figure 12: a) Streamwise evolution of the instantaneous spanwise-averaged components of the disturbance velocity $\overline{\boldsymbol{u}}_{o s}$ and $\overline{\boldsymbol{u}}_{s q}$ in the upper boundary layer at the entrance of the domain.

\section{Analysis of the bypass transition and discussion}

In this section, the disturbance is defined relative to the mean spanwise averaged velocity field depicted in Section 4.2, and the disturbance velocity fields are decomposed so that $\boldsymbol{u}=\boldsymbol{u}_{o s}+\boldsymbol{u}_{s q}$ using the orthogonal decomposition of Section 3.1 .

\subsection{First bypass transition}

As indicated in Section 4 a turbulent transition is first observed on the upper wall near the inlet, characterized by a fast growth of steady streaks, that further experience inflectional secondary instabilities downstream. The streamwise evolution of the velocity components of $\overline{\boldsymbol{u}}_{o s}$ and $\overline{\boldsymbol{u}}_{s q}$ in the upper boundary layer at the entrance of the domain is plotted on Figure 12. As can be seen, the disturbance velocity field contains mainly an SQ streamwise component $u_{s q}$ before the turbulent transition point near $X \approx 8$. The first instability on the upper wall corresponds to large steady streaks generated from the inlet perturbation described in Section 3.3 .

Plots of the instantaneous streamwise velocity in a plane parallel to the wall are shown in Figure $13 \mathrm{a}$ where the streaks and turbulent transition are clearly visible. On this figure, the sinuous instability is clearly seen near $X \approx 8$, whereas the transitional varicose instability is only apparent on the streamwise planes in Figure 13b. The classical regular oscillating structure of the streaks is drawn on top at $X=1$, and corresponds to the linear transient growth phase. At $X=4$, the low speed streaks (in dark on the figure) have a two-lobed mushroom-like shape, enhanced by transient varicose instabilities. This is characteristic of a nonlinear 

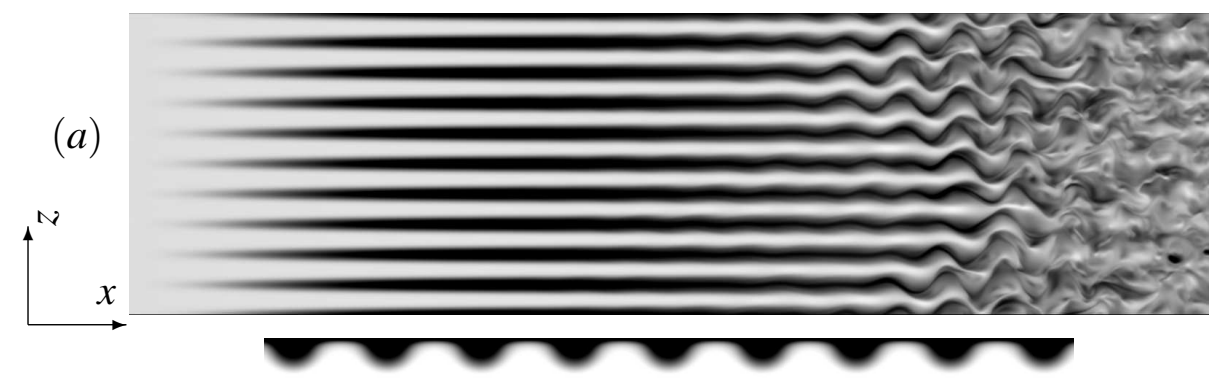

(b)
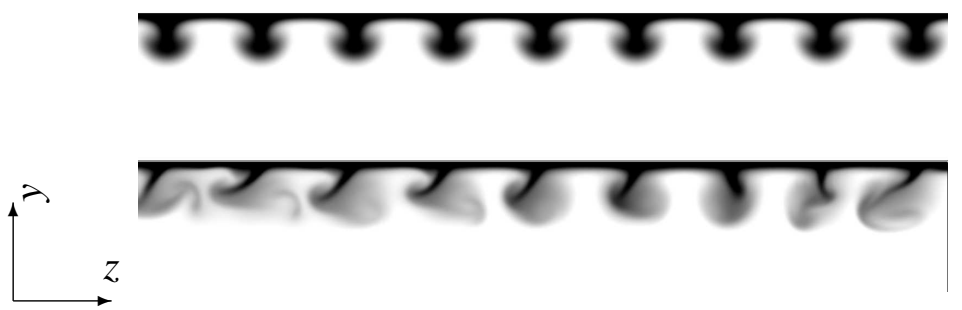

Figure 13: Streak instabilities in the upper boundary layer. Visualization of the instantaneous streamwise velocity $u$ in a plane parallel to the upper wall at $y / h=0.33$ and $0 \leq x / h \leq 10$ a) and in 3 streamwise planes at $X=1,4,9 \mathrm{~b}$ ). The black color corresponds to low speed streaks $\left(u<0.5 U_{0}\right)$ and the white to high speed velocity $\left(u>U_{0}\right)$. 
interaction with the base flow. Further downstream, these mushroom shapes are subjected to sinuous instabilities (Figure 13b at $X=9$ ). They tilt, twist, interact with the wall and the neighboring structures, and then develop into complex forms as they move downstream into the fully turbulent region. .

The transition scenario observed is different from that predicted by previous analyses (Andersson et al. [2], Hoepffner et al. [33], Cossu et al. [21]) on the stability of steady streaks, because, as explained in Section 3.3, the streaks are different (see Figure $4 \mathrm{~b}$ ), and the location of their stability analysis $(x=2 L \approx 20 h)$ (symbol $\boldsymbol{\nabla}$ on Figure $4 \mathrm{~b}$ ) is far downstream from the observed transition point (symbol $\square$ on Figure 4b). Furthermore, their streaky mean profile at $X=20$ (see Figure 13 on page 51 in Andersson et al. [2]) is very different from the mean profile in Figure 7. Thus, their stability analysis is not relevant for the present study.

Varicose and mushroom structures have also been considered by Brandt [9] who studied the instability and transition of an isolated low speed streak numerically, corresponding to the experiment of Asai et al. [4]. By studying the varicose and sinuous instability separately, it was found that the varicose instability is greater close to the location of perturbations, but its growth rate decreases faster downstream, whereas the sinuous instability persists for a larger time. For a random perturbation of the streaks, the two instabilities are in competition, and a first growth of a varicose instability, that is damped further downstream, followed by a sinuous instability can thus be expected as the present simulations shows.

\subsection{Second bypass transition}

The second transition observed on the lower boundary layer at $X \approx 100$ results from the interaction with the upper turbulent boundary layer. It is important to point out here that our simulation is, to the author's knowledge, the first DNS of boundary layer transition induced by "real" external turbulence (i.e. a turbulence generated inside the computational domain). Previous DNS of boundary layer transition induced by free-stream turbulence have used some sort of synthetic turbulence as the inlet boundary conditions (e.g. Brandt et al. [12], Durbin and $\mathrm{Wu}$ [25], Wu and Moin [55]).

As in Section 5.1, the streamwise evolution of the velocity components of $\overline{\boldsymbol{u}}_{O S}$ and $\overline{\boldsymbol{u}}_{s q}$ in the lower boundary layer is plotted near the transition point on Figure 14. Here, we observe the growth of the SQ streamwise component $u_{s q}$, characteristic of the formation of streaks in the boundary layer. Near the transition point, a fast growth of the streamwise component $u_{o s}$ is observed, indicating that 


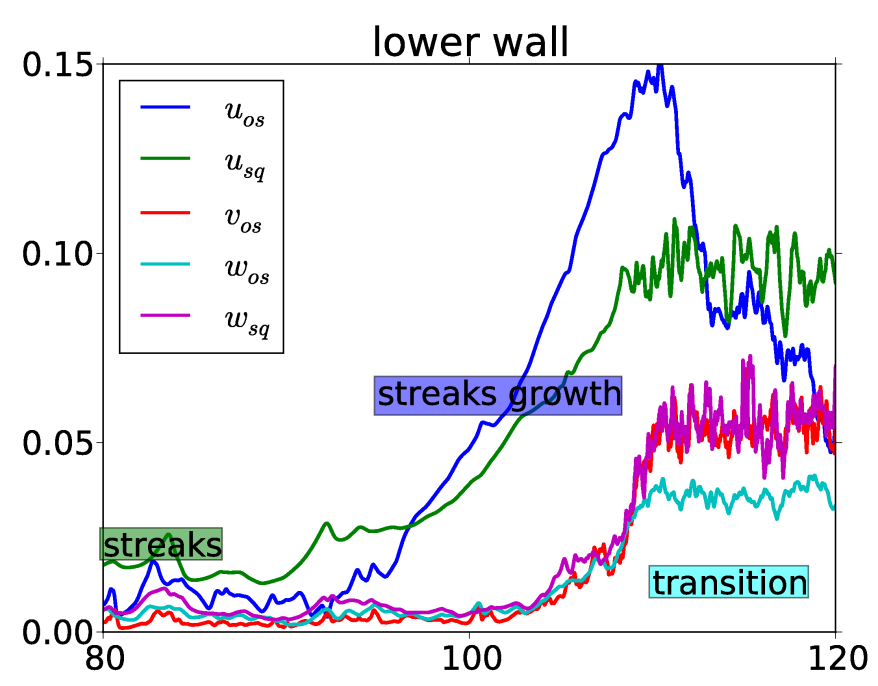

Figure 14: Streamwise evolution of the instantaneous spanwise-averaged components of the disturbance velocity $\overline{\boldsymbol{u}}_{o s}$ and $\overline{\boldsymbol{u}}_{s q}$ in the lower boundary layer near the second transition point at $X \approx 100$.

the streaks are unsteady and wavy. Further downstream, turbulent transition occurs characterized by the fast growth of all the velocity components of $\overline{\boldsymbol{u}}_{o s}$ and $\overline{\boldsymbol{u}}_{s q}$. To analyze the origin of this transition, on Figure [15] we have plotted a the normal OS velocity $v_{o s}$ averaged along the normal direction inside the boundary layer. As can be seen, we observe that large scale oscillations appear in the spanwise direction before the transition (that starts at $X \approx 100$ ). The scale of these oscillations is in the order of the boundary layer thickness $\delta \approx 0.3 h-0.4 h$, and corresponds to the transverse wavenumber $\beta \approx 2 / \delta$, i.e. in the order of magnitude of the optimal perturbation wavenumber. These oscillations of very small amplitude $(\sim 0.4-0.6 \%)$ in the normal velocity $v_{o s}$ profiles induce 10 times larger oscillations in the streamwise velocity, which correspond to streaks, as seen in the profiles of the SQ streamwise velocity $u_{s q}$ plotted on Figure $15 \mathrm{~b}$.

The instantaneous wall pressure disturbance averaged in the spanwise direction is shown on Figure 15b. The high frequency oscillations of the pressure disturbance in the upper boundary layer are characteristic of near-wall turbulence. On the lower wall, before the transition point, only low frequency oscillations are observed. The scale is in the order of the streamwise correlation length scale $L_{11,1} \sim 3 h$ of the streamwise velocity in the plane $y=0$ corresponding to the outer region of the upper turbulent boundary layer. These oscillations reflect the large 

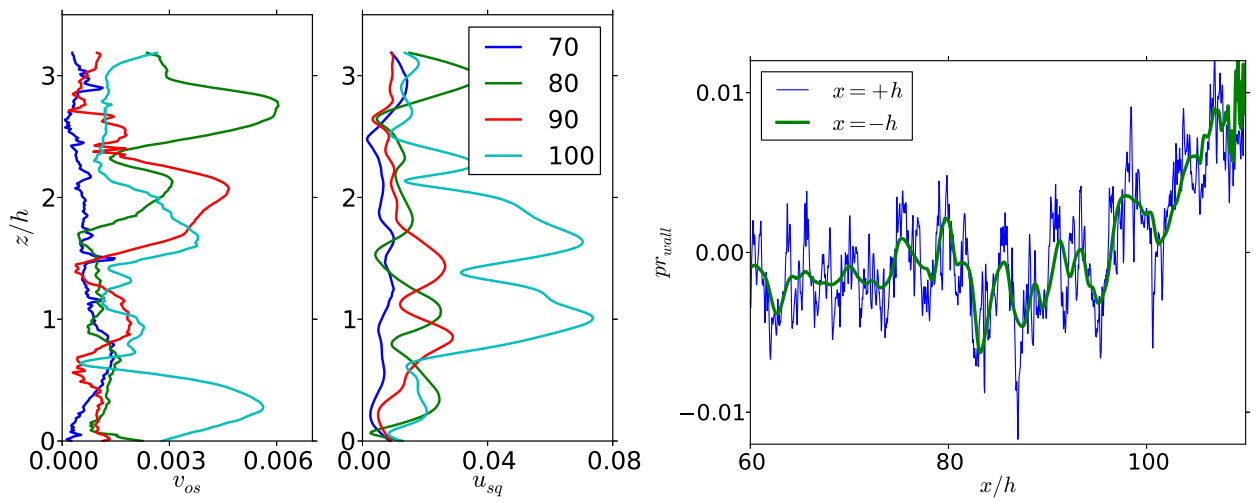

Figure 15: Instantaneous disturbance velocity averaged along the normal direction inside the lower boundary layers at different sections before the transition point: a) OS normal velocity $v_{o s}$ and SQ longitudinal velocity $u_{s q}$, b) wall pressure disturbance averaged in the spanwise direction

scale oscillations of the pressure disturbance in the upper turbulent boundary layer. These low frequency oscillations induce fluctuations in the streamwise velocity that are apparent in the $\bar{u}_{O S}$ profile before the transition point as seen on Figure 14. This indicates that the streaks (spanwise oscillations of the SQ streamwise velocity field $u_{s q}$ ) are modulated in the streamwise direction by the OS streamwise velocity field $u_{o s}$. This mechanism is similar to the formation of non-stationary streaks initiated by two eigenmodes of the Orr-Sommerfeld equations as described by Buffat et al. [15].

On Figure 16, we have plotted the instantaneous streamwise velocity in the lower boundary layer near the transition point at $X \approx 100$. On this figure, the formation of low speed streaks (in black) and their sinuous instabilities are clearly seen in the plane parallel to the wall. The breakdown of the streaks is similar to that described in Section 5.1. As seen on Figure 16b in the plane $X=95$, low speed streak appear in the near-wall region of the boundary layer, due to the liftup mechanism induced by normal velocity oscillations of very small amplitude but with a scale in the order of the boundary layer thickness. Then, non-linear transient growth and transient varicose instabilities push these low speed streaks away from the wall to form two-lobed mushroom-shaped structures as seen in the plane $X=100$. Further downstream, these structures are subject to sinuous instabilities as seen in the plane $X=115$.

In the plane parallel to the wall on Figure 16a, a turbulent spot is observed inside a low speed streak downstream of the fully-turbulent boundary layer. The development over time of this turbulent spot is shown on Figure 17. The spot 

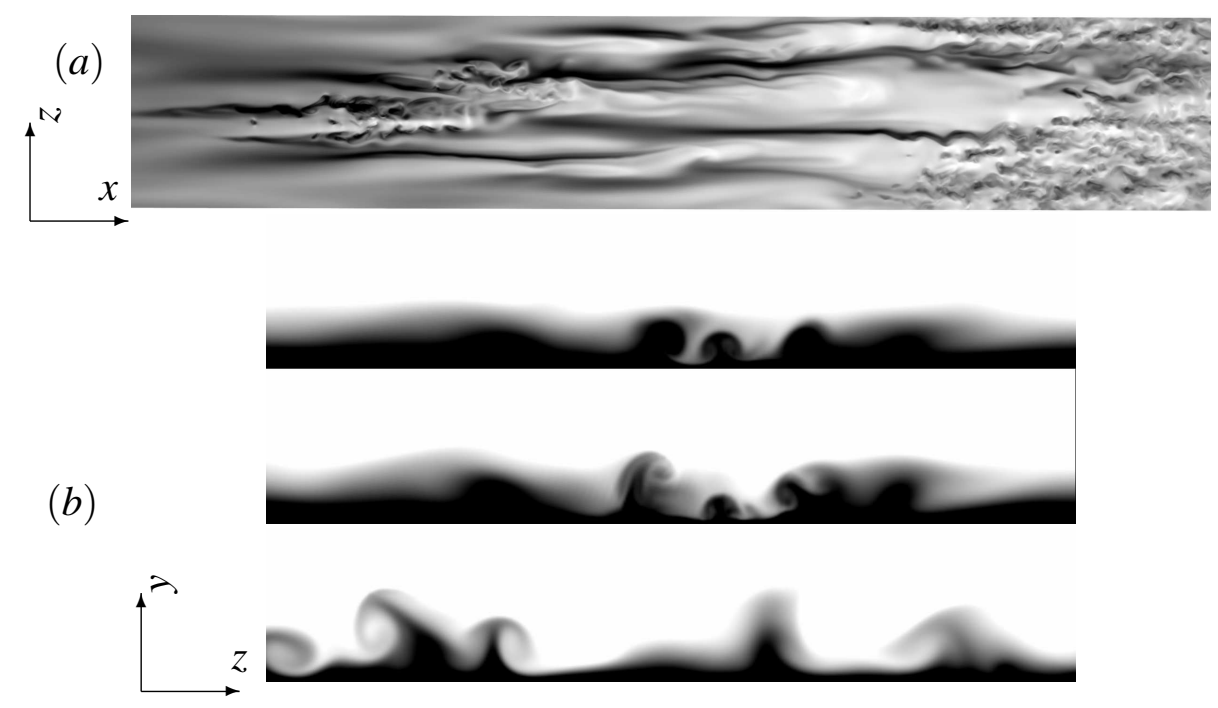

Figure 16: Streak instabilities in the lower boundary layer. Visualization of the instantaneous streamwise velocity $w$ in a plane parallel to the lower wall a) at $y / h=0.15$ and $80 \leq X \leq 120$ and in 3 streamwise planes b) at $X=95,100,115$. The black color corresponds to low speed streaks $\left(u<0.5 U_{0}\right)$ and the white to high speed velocity $\left(u>U_{0}\right)$.

originates initially in a low speed streak, represented by black furrows on the figure. The rear and front part of the disturbance is convected with a mean streaks velocity of $\sim 0.6 U_{e}$ and $\sim 0.9 U_{e}$, respectivelu, where $U_{e}=1.2 U_{0}$ is the outer mean velocity.

To determine the average spacing between the streaks, spanwise correlation functions $R_{u u}$ of streamwise velocity fluctuations are evaluated inside the lower boundary layer. According to the value of the distance of the first minimum (Figure 18), the average streak spacing is equal to $0.9 h$ (double the distance of the first minimum) and corresponds to a streamwise wavenumber $\beta$ close to the wave number of the optimal streaks $\beta=2 / \delta$, where $\delta \sim 0.33 h$ is the mean boundary layer thickness in that region. On Figure 18, contour levels of $R_{u и}$ are also plotted in the middle plane $y=0$, located before the second transition in the outer region of the upper turbulent boundary layer. As can be seen, the correlation function is highly anisotropic with a significant spatial coherence in the streamwise direction. The spanwise width of the positive correlation region is about $\sim 0.4 h$, which corresponds to the streak spacing in the lower boundary layer. The streamwise length of the positive correlation region is about $2 h$, indicating the presence of largescale spatially coherent structures whose streamwise scales are similar to those of the wall pressure disturbances in the lower turbulent boundary layer (Figure 15b). 


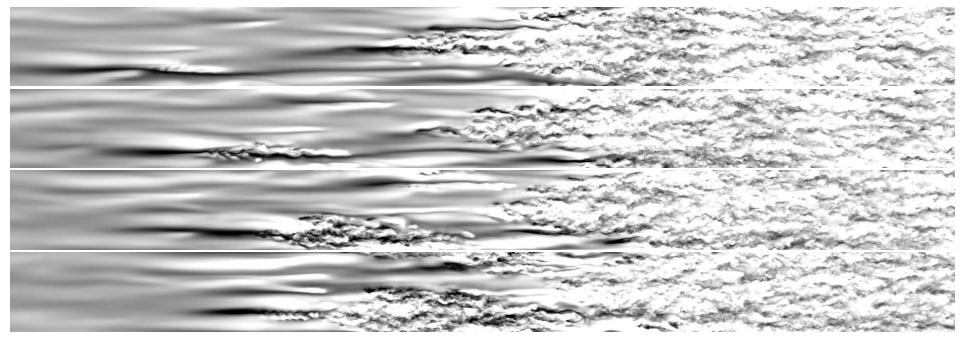

Figure 17: Snapshots of the downstream evolution of a turbulent spot near the second transition with time interval $d t=4.5 \mathrm{~h} / U_{0}$. Levels of the instantaneous streamwise velocity $u$ from black $\left(u<0.5 U_{0}\right)$ to white $\left(u>U_{0}\right)$ in a plane parallel to the wall at $y / h=-0.85$ with $90<X<130$.

The shape of the correlation function is comparable to those measured by PIV in a ZPG turbulent boundary layer (Ganapathisubramani et al. [29]). However, the calculated streamwise extent is much larger than that measured in the wake region of a ZPG turbulent boundary layer, which extends a distance of only $0.6 \delta \approx 0.6 h$. But, as pointed out by Ganapathisubramani et al. [29], the measurements of $R_{u u}$ made by Christensen [19] in a turbulent channel flow show that the channel data exhibit longer streamwise correlations than in a ZPG turbulent boundary layer.

\section{Conclusion}

To conclude, DNS of the boundary layer bypass transition at the entrance of a plane channel has been presented and analyzed. The transition of the upper boundary layer is triggered by a perturbation inside the boundary layer near the entrance of the channel. In the simulations, the perturbation is imposed at the inlet section and consists of the optimal perturbation, $\mathbf{u}^{\text {opt }}$, obtained from the local linear stability theory, with a small turbulence intensity $T u^{o p t} \approx 1.7 \%$ and an additional smaller random vector, $\mathbf{u}^{r}$, with $T u^{r} \approx 0.1 \%$. This perturbation leads to a fast growth of steady streaks, that further break down, leading to the turbulent transition of the upper boundary layer. The transition of the lower boundary layer is induced by the interaction with the upper boundary layer further downstream. This kind of simulations is, to the author's knowledge, the first DNS of boundary layer bypass transition induced by "real" external turbulence generated inside the computational domain. The numerical simulations of such an experiment typically require billions of modes using spectrally accurate approximations and have been calculated using a spectrally-accurate code "NadiaSpectral", specially designed to run on massively parallel high performance computers (Montagnier et al. [42]). 

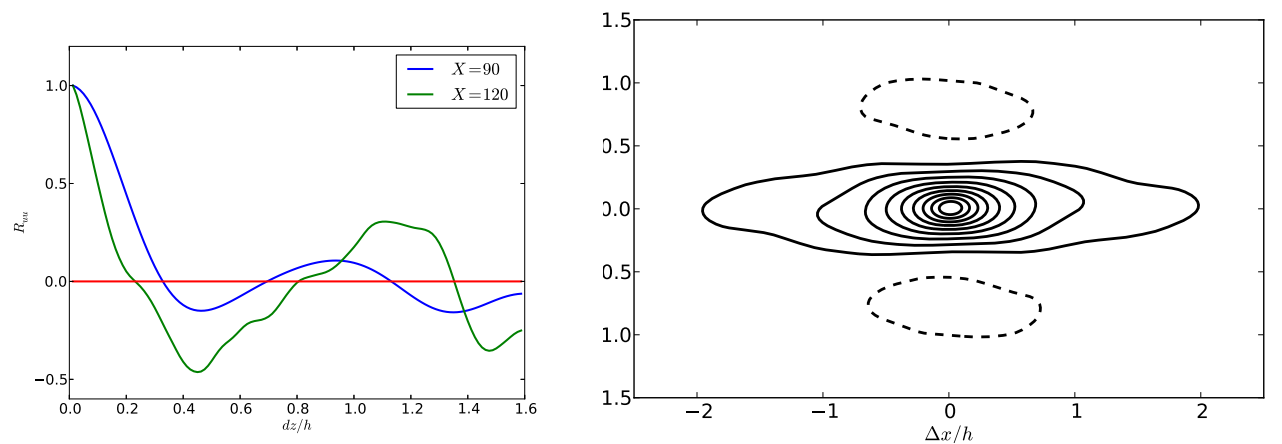

Figure 18: Spanwise correlation functions $R_{u u}$ of the streamwise velocity fluctuations. a) Spanwise profiles of $R_{u u}$ inside the lower boundary layer at different sections X. b) Contour levels of $R_{u u}$ in the middle plane $y=0$ between $X=60$ and $X=100$. The contour levels for $R_{u u}$ range from -0.1 to 1.0 with spacing of 0.1 . Zero contours are not shown. 
For the turbulent upper boundary layer, the self similar logarithmic law of the wall has been recovered. In the outer region, the profiles are also self-similar but differ significantly from the ZPG boundary layer results because of the small favorable pressure gradient in the channel. Further downstream, low frequency fluctuations induced by the turbulent upper boundary layer generate streaks in the near-wall region of the lower laminar boundary layer. Then, non-linear breakdown of these streaks induces a bypass transition of the lower boundary layer. Afterwards, turbulence occupies the whole channel width and a turbulent channel flow develops.

To analyze the transition, an orthogonal decomposition of solenoidal velocity fields is used on the disturbance flow. The velocity perturbation is expressed as an $L_{2}$ orthogonal sum of an Orr Sommerfeld velocity field (function of the perturbation normal velocity) and a Squire velocity field (function of the perturbation normal vorticity). Using this orthogonal decomposition, the streaks are characterized as an SQ velocity field, with a large streamwise component $u_{s q}$, which could be modulated in the streamwise direction by an OS velocity field independent of the spanwise direction. The SQ velocity field experiences strong non-linear growth that pushes low speed streaks away from the wall and forms mushroom-shaped structures that are subject to transient varicose instabilities in the wall-normal direction. Further downstream, the heads of these low speed streaks are subject to sinuous instabilities in the spanwise direction, followed by a breakdown and a turbulent transition. This scenario has been confirmed by animated views of the results that show the formation and breakdown of the streaks, which are provided on video in Buffat [13]. Recent studies at lower Reynolds number Re (Buffat et al. [16]), corresponding to a smaller channel height, show that these varicose instabilities can become strong enough to induce Kelvin-Helmholtz instabilities at the top of the low speed streaks and a turbulent transition is observed without the development of sinuous instabilities. The transition scenario observed is different from previous analyses on the bypass transition of steady streaks in a ZPG boundary layer (Andersson et al. [2], Brandt et al. [10], Cossu et al. [21]). The transitional structures observed are however similar to the mushroom-shaped structures obtained by Bernard [5] in his simulations of ZPG transitional boundary layers. Beyond these considerations, there may be advantages in investigating parametric studies for different Reynolds $R e_{h}$ and different levels of inlet perturbation. These and similar considerations will be pursued in further studies. 


\section{Acknowledgments}

The authors would like to thank the P2CHPD computer center at the Université Claude Bernard Lyon 1, member of the Fédération Lyonnaise de Modélisation et Sciences Numériques 1 , for providing the computer facilities. This work was also done using HPC resources from GENCI at IDRIS in France and the PRACE Research Infrastructure resource JUGENE based in Germany.

\section{Bibliography}

[1] Andersson, P., Berggren, M., Henningson, D. S., 1999. Optimal disturbances and bypass transition in boundary layers. Physics of Fluids 11, 134-150.

[2] Andersson, P., Brandt, L., Bottaro, A., Henningson, D. S., 2001. On the breakdown of boundary layers streaks. J. Fluid Mech. 428, 29-60.

[3] Asai, M., Floryan, J. M., 2003. Certain aspects of channel entrance flow. Phys. Fluids 16 (4), 1160-1163.

[4] Asai, M., Minagawa, M., Nishioka, M., 2002. The instability and breakdown of a near-wall low-speed streak. J. Fluid Mech. 445.

[5] Bernard, P. S., 2011. The hairpin vortex illusion. Journal of Physics: Conference Series, ETC13 318 (6), 062004.

[6] Bertolotti, F. P., Herbert, T., Spalard, P. R., 1992. Linear and nonlinear stability of the blasius boundary layer. Journal of Fluid Mechanics 242, 441-474.

[7] Bertolotti, F. P., Herbert, T., Spalart, P. R., 1992. Linear and nonlinear stability of the Blasius boundary layer. J. Fluid Mech. 242, 441-474.

[8] Biau, D., Soueid, H., Bottaro, A., 2008. Transition to turbulence in duct flow. Journal of Fluid Mechanics 596, 133-142.

[9] Brandt, L., 2007. Numerical studies of the instability and breakdown of a boundary-layer low-speed streak. European Journal of Mechanics B/Fluids 26, 64-82.

\footnotetext{
1 http://www.flmsn.univ-lyon1.fr
} 
[10] Brandt, L., Cossu, C., Chomaz, J.-M., Huerre, P., Henningson, D. S., 2003. On the convectively unstable nature of optimal streaks in boundary layers. Journal of Fluid Mechanics 485, 221-242.

[11] Brandt, L., Henningson, D., 2002. Transition of streamwise streaks in zeropressure-gradient boundary layers. J. Fluid Mech. 472, 229-262.

[12] Brandt, L., Schlatter, P., Henningson, D. S., 2004. Transition in boundary layers subject to free-stream turbulence. Journal of Fluid Mechanics 517, 167-198.

[13] Buffat, M., 2008. NadiaSpectral: a DNS tools for wall bounded flow 'http://www.ufrmeca.univ-lyon1.fr/\{ \}buffat/Nadiaspectral'.

[14] Buffat, M., Le Penven, L., May 2013. Analysis of transient growth using an orthogonal decomposition of the velocity field in the Orr-Sommerfeld Squire equations. ArXiv e-prints.

[15] Buffat, M., Le Penven, L., Cadiou, A., March 2011. An efficient spectral method based on an orthogonal decomposition of the velocity for transition analysis in wall bounded flow. Computers \& Fluids 42, 62-72.

[16] Buffat, M., Le Penven, L., Cadiou, A., 2013. Non modal subcritical transition of channel entry flow. In: 14th Europen Turbulence Conference, Lyon, September 1-4.

[17] Butler, K. M., Farrell, B. F., 1992. Three-dimensional optimal perturbations in viscous shear flow. Phys. Fluids A 4, 1637-1650.

[18] Chen, T. S., Sparrow, E. M., 1967. Stability of the developing laminar flow in a parallel-plate channel. Journal of Fluid Mechanics 30, 2, 209-224.

[19] Christensen, K. T., Dec. 2001. Experimental investigation of acceleration and velocity fields in turbulent channel flow. Ph.D. thesis, University of Illinois at Urbana-Champaign. URL http://adsabs.harvard.edu/abs/2001PhDT......86C

[20] Corbett, P., Bottaro, A., 2000. Optimal perturbations for boundary layers subject to stream-wise pressure gradient. Phys. Fluids 12, 120-130. 
[21] Cossu, C., Brandt, L., Bagheri, S., Henningson, D. S., 2011. Secondary threshold amplitudes for sinuous streak breakdown. Physics of Fluids 23, 074103 .

[22] Criminale, W. O., Jackson, T. L., Joslin, R. D., 2003. Theory and computation of hydrodynamic stability. Cambridge University Press.

[23] Dean, R. B., Dec. 1978. Reynolds number dependence of skin friction and other bulk flow variables in two-dimensional rectangular duct flow. Journal of Fluid Engineering 100, 215-222.

[24] Duck, P. W., 2005. Transient growth in developing plane and Hagen Poiseuille flow. Proc. R. Soc. A 461, 1311-1333.

[25] Durbin, P., Wu, X., 2007. Transition beneath vortical disturbances. Annu. Rev. Fluid Mech. 39, 107-128.

[26] Durst, F., Ray, S., Unsal, B., Bayoumi, O. A., 2005. The development lengths of laminar pipe and channel flows. Journal of Fluids Engineering 127, 1154-1160.

[27] Fransson, J. H. M., Brandt, L., Talamelli, A., Cossu, C., 2004. Experimental and theoretical investigation of the nonmodal growth of steady streaks in a flat plate boundary layer. Physics of Fluids 16,10, 3627-3638.

[28] Fransson, J. H. M., Talamelli, A., 2012. On the generation of steady streamwise streaks in flat-plate boundary layers. Journal of Fluid Mechanics 698, 211-234.

[29] Ganapathisubramani, B., Hutchins, N., Hambleton, W. T., Longmire, E. K., Marusic, I., Feb. 2005. Investigation of large-scale coherence in a turbulent boundary layer using two-point correlations. Journal of Fluid Mechanics 524 (2005), 57-80. URL http://www.journals.cambridge.org/abstract_s0022112004002277

[30] Gustavsson, L. H., 1991. Energy growth of three-dimensional disturbances in plane Poiseuille flow. J. fluid Mech. 224, 241-260.

[31] Gustavsson, L. H. \& Hultgren, L. S., 1980. A resonance mechanism in plane Couette flow. J. Fluid Mech. 98, 149-159. 
[32] Hifdi, A., Touhami, M. O., Naciri, J. K., 2004. Channel entrance flow and its linear stability. Journal of Statistical Mechanics: Theory and Experiment, P06003.

[33] Hoepffner, J., Brandt, L., Henningson, D. S., 2005. Transient growth on boundary layer streaks. J. Fluid Mech. 537, 91-100.

[34] Jacobs, R. G., Durbin, P. A., 2001. Simulations of bypass transition. J. Fluid Mech 428, 185-212.

[35] Levin, O., Henningson, D. S., 2003. Exponential vs algebraic growth and transition prediction in boundary layer flow. Flow, Turbul. Combust 70, 183.

[36] Lien, K., Monty, J. P., Chong, M. S., Ooi, A., 2004. The entrance length for fully developed turbulent channel flow. In: 15th Australasian Fluid Mechanics Conference The University of Sydney, Sydney, Australia.

[37] Luchini, P., 1996. Reynolds-number-independent instability of the boundary layer over a flat surface. Journal of Fluid Mechanics 327, 101-115.

[38] Luchini, P., 2000. Reynolds-number-independent instability of the boundary layer over a flat surface: optimal perturbations. Journal of Fluid Mechanics 404, 289-309.

[39] Lund, T. S., Wu, X., Squires, K. D., 1998. Generation of turbulent inflow data for spatially-developing boundary layer simulations. Journal Of Computational Physics 140, 233-258.

[40] Matsubara, M., Alfredsson, P. H., 2001. Disturbance growth in boundary layers subjected to free-stream turbulence. Journal of Fluid Mechanics 430, $149-168$.

[41] Monkewitz, P. A., Chauhan, K. A., Nagib, H. M., 2007. Self-consistent highreynolds-number asymptotics for zero-pressure gradient turbulent boundary layer. Physics of Fluids 19, 115101.

[42] Montagnier, J., Cadiou, A., Buffat, M., Penven, L. L., 2012. Towards petascale simulation for transition analysis in wall bounded flow. International Journal for Numerical Methods in Fluids.

[43] Moser, R. D., Kim, J., Mansour, N. N., 1999. Direct numerical simulation of turbulent channel flow up to ret=590. Phys. Fluids 11, 943-945. 
[44] Mullin, T., 2011. Experimental studies of transition to turbulence in a pipe. Annual Review of Fluid Mechanics 43 (1), 1-24.

[45] Orszag, S., 1971. Accurate solution of the Orr-Sommerfeld stability equation. J. Fluid Mech. 50, 689-703.

[46] Roach, P. E., Brierley, D. H., 1992. The influence of a turbulent free-stream on zero pressure gradient transitional boundary layer development. Part I: Test cases T3A and T3B. In: Pironneau, O., Rodi, W., Ryhming, I., Savill, A., Truong, T. (Eds.), Numerical simulation of unsteady flows and transition to turbulence. Cambridge University Press,, pp. 303-316.

[47] Sadri, R., Floryan, J., 2002. Entry flow in a channel. Computers and Fluids 31, 133-157.

[48] Schlatter, P., Örlü, R., Li, Q., Brethouwer, G., Johansson, A. V., Alfredsson, P. H., Henningson, D. S., 2011. Progress in simulations of turbulent boundary layers. In: 7th International Symposium on Turbulence and Shear Flow Phenomena, 28-31 July 2011, Ottawa, Canada.

[49] Schmid, P. J., Henningson, D. S., 2001. Stability and Transition in Shear Flows. Springer.

[50] Shah, R. K., London, A. L., 1978. Laminar flow forced convection in ducts: a source book for compact heat exchanger analytical data. Academic Press, New York.

[51] Simens, M. P., Jiménez, J., Hoyas, S., Mizuno, Y., 2009. A high-resolution code for turbulent boundary layers. J. Comp. Phys. 228, 4218-4231.

[52] Sparrow, E., Lin, S. H., Lundgren, T. S., 1964. Flow development in the hydrodynamaic entrance region of tubes and ducts. Physics of Fluids 7, 3, 338-347.

[53] Trefethen, L. N., Trefethen, A. E., Reddy, S. C., Driscoll, T. A., 1993. Hydrodynamic stability without eigenvalues. Science 261, 578-584.

[54] Tumin, A., Reshotko, E., Jul. 2001. Spatial theory of optimal disturbances in boundary layers. Phys. Fluids 13 (7), 2097-2104. 
[55] Wu, X., Moin, P., 2009. Direct numerical simulation of turbulence in a nominally zero-pressure-gradient flat-plate boundary layer. Journal of Fluid Mechanics $630,5-41$.

[56] Zaki, T. A., Durbin, P. A., 2005. Mode interaction and the bypass route to transition. J. Fluid Mech. 85-111. 